\title{
LYUBEZNIK NUMBERS OF LOCAL RINGS AND LINEAR STRANDS OF GRADED IDEALS
}

\author{
JOSEP ÀLVAREZ MONTANER AND KOHJI YANAGAWA
}

\begin{abstract}
In this work we introduce a new set of invariants associated to the linear strands of a minimal free resolution of a $\mathbb{Z}$-graded ideal $I \subseteq R=\mathbb{k}\left[x_{1}, \ldots, x_{n}\right]$. We also prove that these invariants satisfy some properties analogous to those of Lyubeznik numbers of local rings. In particular, they satisfy a consecutiveness property that we prove first for the Lyubeznik table. For the case of squarefree monomial ideals we get more insight on the relation between Lyubeznik numbers and the linear strands of their associated Alexander dual ideals. Finally, we prove that Lyubeznik numbers of StanleyReisner rings are not only an algebraic invariant but also a topological invariant, meaning that they depend on the homeomorphic class of the geometric realization of the associated simplicial complex and the characteristic of the base field.
\end{abstract}

\section{INTRODUCTION}

Let $A$ be a noetherian local ring that admits a surjection from an $n$-dimensional regular local ring $(R, \mathfrak{m})$ containing its residue field $\mathbb{k}$, and $I \subseteq R$ be the kernel of the surjection. In [13], Lyubeznik introduced a new set of invariants $\lambda_{p, i}(A)$ as the $p$-th Bass number of the local cohomology module $H_{I}^{n-i}(R)$, that is

$$
\lambda_{p, i}(A):=\mu^{p}\left(\mathfrak{m}, H_{I}^{n-i}(R)\right)=\operatorname{dim}_{\mathbb{k}} \operatorname{Ext}_{R}^{p}\left(\mathbb{k}, H_{I}^{n-i}(R)\right)
$$

and they depend only on $A, i$ and $p$, but not on the choice of $R$ or the surjection $R \longrightarrow A$. In the seminal works of Huneke-Sharp [10] and Lyubeznik [13] it is proven that these Bass numbers are all finite. Denote $d=\operatorname{dim} A$, Lyubeznik numbers satisfy the following properties]:

i) $\lambda_{p, i}(A) \neq 0$ implies $0 \leq p \leq i \leq d$.

ii) $\lambda_{d, d}(A) \neq 0$.

iii) Euler characteristic:

$$
\sum_{0 \leq p, i \leq d}(-1)^{p-i} \lambda_{p, i}(A)=1
$$

The first author was partially supported by Generalitat de Catalunya 2014SGR-634 project and Spanish Ministerio de Economía y Competitividad MTM2015-69135-P. The second author was partially supported by JSPS KAKENHI 25400057.

${ }^{1}$ Property iii) was shown to us by R. García-López (see [2] for details). 
Therefore, we can collect them in the so-called Lyubeznik table:

$$
\Lambda(A)=\left(\begin{array}{ccc}
\lambda_{0,0} & \cdots & \lambda_{0, d} \\
& \ddots & \vdots \\
& & \lambda_{d, d}
\end{array}\right)
$$

and we say that the Lyubeznik table is trivial if $\lambda_{d, d}=1$ and the rest of these invariants vanish.

Despite its algebraic nature, Lyubeznik numbers also provide some geometrical and topological information as it was already pointed out in [13]. For instance, in the case of isolated singularities, Lyubeznik numbers can be described in terms of certain singular cohomology groups in characteristic zero (see [6]) or étale cohomology groups in positive characteristic (see [5], 4]). The highest Lyubeznik number $\lambda_{d, d}(A)$ can be described using the so-called Hochster-Huneke graph as it has been proved in [15], [31. However very little is known about the possible configurations of Lyubeznik tables except for low dimension cases [12, 24] or the just mentioned case of isolated singularities.

In Section 2 we will give some new constraints to the possible configurations of Lyubeznik tables. Namely, the main result, Theorem 2.1, establishes some consecutiveness of the non-vanishing superdiagonals of the Lyubeznik tables using spectral sequence arguments.

In Section 3 we introduce a new set of invariants associated to the linear strands of a minimal free resolution of a $\mathbb{Z}$-graded ideal $I \subseteq R=\mathbb{k}\left[x_{1}, \ldots, x_{n}\right]$. It turns out that these new invariants satisfy some analogous properties to those of Lyubeznik numbers including the aforementioned consecutiveness property. Moreover, we provide a Thom-Sebastiani type formula for these invariants that is a refinement of the formula for Betti numbers given by Jacques and Katzman in [11. This section should be of independent interest and we hope it could be further developed in future work.

In the rest of the paper we treat the case where $I$ is a monomial ideal in a polynomial ring $R=\mathbb{k}\left[x_{1}, \ldots, x_{n}\right]$, and $\mathfrak{m}=\left(x_{1}, \ldots, x_{n}\right)$ is the graded maximal ideal. Bass numbers are invariant with respect to completion so we consider $\lambda_{p, i}(R / I)=\lambda_{p, i}(\widehat{R} / I \widehat{R})$ where $\widehat{R}=\mathbb{k} \llbracket x_{1}, \ldots, x_{n} \rrbracket$. In this sense, our study on the Lyubeznik tables of monomial ideals is a (very) special case of that for local rings. However, advanced techniques in combinatorial commutative algebra are very effective in this setting, and we can go much further than general case, so we hope that monomial ideals are good "test cases" for the study of Lyubeznik tables.

Since local cohomology modules satisfy $H_{I}^{i}(R) \cong H_{\sqrt{I}}^{i}(R)$ we often will assume that a monomial ideal $I$ is squarefree, i.e., $I=\sqrt{I}$. In this case, $I$ coincides with the StanleyReisner ideal $I_{\Delta}$ of a simplicial complex $\Delta \subseteq 2^{\{1, \ldots, n\}}$, more precisely,

$$
I=I_{\Delta}:=\left(\prod_{i \in F} x_{i} \mid F \subseteq\{1, \ldots, n\}, F \notin \Delta\right) .
$$


The Stanley-Reisner ring $R / I_{\Delta}$ is one of the most fundamental tools in combinatorial commutative algebra, and it is known that $R / I_{\Delta}$ reflects topological properties of the geometric realization $|\Delta|$ of $\Delta$ in several ways.

In Section 4 we get a deeper insight to the relation, given by the first author and A. Vahidi in [1], between Lyubeznik numbers of monomial ideals and the linear strands of their associated Alexander dual ideals. In particular, we give a different approach to the fact proved in [2] that if $R / I_{\Delta}$ is sequentially Cohen-Macaulay, then its Lyubeznik table is trivial. We also provide a Thom-Sebastiani type formula for Lyubeznik numbers.

One of the main results of this paper is left for Section 5. Namely, Theorem 5.3 states that Lyubeznik numbers of Stanley-Reisner rings are not only an algebraic invariant but also a topological invariant, meaning that the Lyubeznik numbers of $R / I_{\Delta}$ depend on the homeomorphic class of the geometric realization $|\Delta|$ of $\Delta$ and the characteristic of the base field.

The proof of this result is quite technical and irrelevant to the other parts of the paper, so we decided to put it in the final section. We also remark that this result holds in a wider setting. More precisely, if $R$ is a normal simplicial semigroup ring which is Gorenstein, and $I$ is a monomial ideal, then the corresponding result holds. We will work in this general setting, since the proof is the same as in the polynomial ring case.

\section{Consecutiveness of nontrivial superdiagonals of the LyubezniK table}

To give a full description of the possible configurations of Lyubeznik tables of any local ring seems to be a very difficult task and only a few results can be found in the literature. The aim of this section is to give some constraints to the possible configurations of Lyubeznik tables, aside from the Euler characteristic formula.

Let $(R, \mathfrak{m})$ be a regular local ring of dimension $n$ containing its residue field $\mathbb{k}$, and $I \subseteq R$ be any ideal with $\operatorname{dim} R / I=d$. For each $j \in \mathbb{N}$ with $0 \leq j \leq d$, set

$$
\rho_{j}(R / I)=\sum_{i=0}^{d-j} \lambda_{i, i+j}(R / I) .
$$

For example, $\rho_{0}(R / I)$ (resp. $\rho_{1}(R / I)$ ) is the sum of the entries in the diagonal (resp. superdiagonal) of the Lyubeznik table $\Lambda(R / I)$. Clearly, $\sum_{j \in \mathbb{N}}(-1)^{j} \rho_{j}(R / I)=1$. We say $\rho_{j}(R / I)$ is non-trivial, if

$$
\rho_{j}(R / I) \geq \begin{cases}2 & \text { if } j=0 \\ 1 & \text { if } j \geq 1\end{cases}
$$

Clearly, $\Lambda(R / I)$ is non-trivial if and only if $\rho_{j}(R / I)$ is non-trivial for some $j$.

It is easy to see that $\lambda_{0, d}(R / I)=0$ if $d \geq 1$ and $\lambda_{0, d}(R / I)=1$ if $d=0$, that is, $\rho_{d}(R / I)$ is always trivial.

A key fact that we are going to use in this section is that local cohomology modules have a natural structure over the ring of $k$-linear differential operators $D_{R \mid k}$ (see [13], [14]). 
In fact they are $D_{R \mid k}$-modules of finite length (see [3, Thm. 2.7.13] and [13, Ex.2.2] for the case of characteristic zero and [14, Thm. 5.7] in positive characteristic). In particular, Lyubeznik numbers are nothing but the length as a $D_{R \mid k}$-module of the local cohomology modules $H_{\mathfrak{m}}^{p}\left(H_{I}^{n-i}(R)\right)$, i.e.

$$
\lambda_{p, i}(R / I)=\operatorname{length}_{D_{R \mid k}}\left(H_{\mathfrak{m}}^{p}\left(H_{I}^{n-i}(R)\right)\right) .
$$

The $D_{R \mid k}$-module length, that will be denoted simply as $e(-)$, is an additive function, i.e given a short exact sequence of holonomic $D_{R \mid k}$-modules $0 \longrightarrow M_{1} \longrightarrow M_{2} \longrightarrow M_{3} \longrightarrow 0$ we have

$$
e\left(M_{2}\right)=e\left(M_{1}\right)+e\left(M_{3}\right)
$$

The main result of this section is the following:

Theorem 2.1. Let $(R, \mathfrak{m})$ be a regular local ring of dimension $n$ containing its residue field $\mathbb{k}$, and $I \subseteq R$ be any ideal with $\operatorname{dim} R / I=d$. Then:

- If $\rho_{j}(R / I)$ is non-trivial for some $j$ with $0<j<d$, then either $\rho_{j-1}(R / I)$ or $\rho_{j+1}(R / I)$ is non-trivial.

- If $\rho_{0}(R / I)$ is non-trivial, then so is $\rho_{1}(R / I)$.

Proof. Consider Grothendieck's spectral sequence

$$
E_{2}^{p, n-i}=H_{\mathfrak{m}}^{p}\left(H_{I}^{n-i}(R)\right) \Longrightarrow H_{\mathfrak{m}}^{p+n-i}(R) .
$$

This is a spectral sequence of $D_{R \mid k^{-}}$modules where $\lambda_{p, i}(R / I)=e\left(E_{2}^{p, n-i}\right)$. Notice also that the local cohomology modules $H_{\mathfrak{m}}^{r}(R)$ vanish for all $r \neq n$ and in this case $e\left(H_{\mathfrak{m}}^{n}(R)\right)=1$.

We will prove the assertion by contradiction. So assume that $\rho_{j}(R / I)$ is non-trivial for some $0<j<d$, but both $\rho_{j-1}(R / I)$ and $\rho_{j+1}(R / I)$ are trivial (the case $j=0$ can be proved by a similar argument). We have some $p, i$ with $i=p+j$ such that $\lambda_{p, i}(R / I) \neq 0$ (equivalently, $E_{2}^{p, n-i} \neq 0$ ). Consider the maps on $E_{2}$-terms

$$
E_{2}^{p-2, n-i+1} \stackrel{d_{2}}{\longrightarrow} E_{2}^{p, n-i} \stackrel{d_{2}^{\prime}}{\longrightarrow} E_{2}^{p+2, n-i-1} .
$$

We will show that $d_{2}=d_{2}^{\prime}=0$.

Consider first the case $j>1$. We have $E_{2}^{p-2, n-i+1}=E_{2}^{p+2, n-i-1}=0$ just because $e\left(E_{2}^{p-2, n-i+1}\right)=\lambda_{p-2, i-1}(R / I)$ and $e\left(E_{2}^{p+2, n-i-1}\right)=\lambda_{p+2, i+1}(R / I)$ concern $\rho_{j+1}(R / I)$ and $\rho_{j-1}(R / I)$ respectively. Therefore $d_{2}=d_{2}^{\prime}=0$ is satisfied trivially. When $j=1$, i.e. the case when $(p+2, n-i-1)=(d, n-d)$, we have:

$$
E_{2}^{d-4, n-d+2} \stackrel{d_{2}}{\longrightarrow} E_{2}^{d-2, n-d+1} \stackrel{d_{2}^{\prime}}{\longrightarrow} E_{2}^{d, n-d} .
$$

The triviality of $\rho_{2}(R / I)$ and $\rho_{0}(R / I)$ means that $E_{2}^{d-4, n-d+2}=0$ and $\lambda_{d, d}=e\left(E_{2}^{d, n-d}\right)=1$ so $d_{2}=0$. Now we assume that the map $d_{2}^{\prime}: E_{2}^{d-2, n-d+1} \rightarrow E_{2}^{d, n-d}$ is non-zero. Then $\operatorname{Im} d_{2}^{\prime}=E_{2}^{d, n-d}$ due to the fact that $E_{2}^{d, n-d}$ is a simple $D_{R \mid k}$-module. It follows that $E_{3}^{d, n-d}=E_{2}^{d, n-d} / \operatorname{Im} d_{2}^{\prime}=0$ so

$$
0=E_{3}^{d, n-d}=E_{4}^{d, n-d}=\cdots=E_{\infty}^{d, n-d} .
$$


On the other hand, since $\rho_{0}(R / I)$ is trivial, we have

$$
0=E_{2}^{i, n-i}=E_{3}^{i, n-i}=\cdots=E_{\infty}^{i, n-i}
$$

for all $i<d$. Therefore we get a contradiction since, by the general theory of spectral sequences, there exists a filtration

$$
0 \subseteq \mathcal{F}_{n}^{n} \subseteq \cdots \subseteq \mathcal{F}_{1}^{n} \subseteq H_{\mathfrak{m}}^{n}(R),
$$

where $E_{\infty}^{i, n-i}=\mathcal{F}_{i}^{n} / \mathcal{F}_{i+1}^{n}$.

Anyway, we have shown that $d_{2}=d_{2}^{\prime}=0$ in all cases, and this implies that $E_{3}^{p, n-i}=$ $E_{2}^{p, n-i} \neq 0$. Now we consider the maps on $E_{3}$-terms

$$
E_{3}^{p-3, n-i+2} \stackrel{d_{3}}{\longrightarrow} E_{3}^{p, n-i} \stackrel{d_{3}^{\prime}}{\longrightarrow} E_{3}^{p+3, n-i-2} .
$$

Since $E_{3}^{p-3, n-i+2}$ and $E_{3}^{p+3, n-i-2}$ concern $\rho_{j+1}(R / I)$ and $\rho_{j-1}(R / I)$ respectively, we have $d_{3}=d_{3}^{\prime}=0$ by the same argument as above. Hence we have $E_{4}^{p, n-i}=E_{3}^{p, n-i} \neq 0$. Repeating this argument, we have $0 \neq E_{2}^{p, n-i}=E_{3}^{p, n-i}=\cdots=E_{\infty}^{p, n-i}$ so we get a contradiction with the fact that $H_{\mathfrak{m}}^{p+n-i}(R)=0$ (recall that $j=i-p \neq 0$ ).

The behavior of the consecutive superdiagonals is reflected in the following example.

Example 2.2. Let $I \subseteq R=\mathbb{k} \llbracket x_{1}, \ldots, x_{8} \rrbracket$ be the Alexander dual ideal of the edge ideal of an 8-cycle, i.e. $I^{\vee}=\left(x_{1} x_{2}, x_{2} x_{3}, \ldots, x_{7} x_{8}, x_{8} x_{1}\right)$. Using the results of [1] we get the Lyubeznik table

$$
\Lambda(R / I)=\left(\begin{array}{lllllll}
0 & 0 & 0 & 0 & 1 & 0 & 0 \\
& 0 & 0 & 0 & 0 & 0 & 0 \\
& & 0 & 0 & 0 & 1 & 0 \\
& & & 0 & 0 & 1 & 0 \\
& & & & 0 & 0 & 0 \\
& & & & & 0 & 1 \\
& & & & & & \\
& & & & & & 1
\end{array}\right) .
$$

Notice that $\rho_{0}(R / I)$ being trivial does not imply that $\rho_{1}(R / I)=0$.

Remark 2.3. Using similar spectral sequence arguments to those considered in Theorem 2.1, Kawasaki [12] and Walther 24] described the possible Lyubeznik tables for rings up to dimension two. Namely, their result is:

- If $d=2$, then $\lambda_{2,2}(R / I)-1=\lambda_{0,1}(R / I)$ and the other Lyubeznik numbers are 0 .

If we take a careful look at the spectral sequence we can also obtain the following:

- If $d \geq 3$, then $\lambda_{2, d}(R / I)=\lambda_{0, d-1}(R / I)$ and

$$
\begin{aligned}
\lambda_{1, d-1}(R / I) \leq \lambda_{3, d}(R / I) & \leq \lambda_{1, d-1}(R / I)+\lambda_{0, d-2}(R / I) \\
& \leq \lambda_{3, d}(R / I)+\lambda_{2, d-1}(R / I) .
\end{aligned}
$$

For $d=3$ we can refine the last inequality, that is,

$$
\lambda_{1,2}(R / I)+\lambda_{0,1}(R / I)=\lambda_{3,3}(R / I)+\lambda_{2,2}(R / I)-1 .
$$


Indeed, using the filtration (2.1) we have

$$
\sum_{i=0}^{d} e\left(E_{\infty}^{i, n-r-i}\right)=e\left(H_{\mathfrak{m}}^{n-r}(R)\right)= \begin{cases}1 & \text { if } r=0 \\ 0 & \text { otherwise. }\end{cases}
$$

Then the result follows considering the differentials $d_{2}: E_{2}^{0, n-d+1} \longrightarrow E_{2}^{2, n-d}, d_{2}$ : $E_{2}^{1, n-d+1} \longrightarrow E_{2}^{3, n-d}, d_{2}: E_{2}^{0, n-d+2} \longrightarrow E_{2}^{2, n-d+1}$ and $d_{3}: E_{3}^{0, n-d+2} \longrightarrow E_{3}^{3, n-d}$. Finally, we point out that $E_{3}^{0, n-d+1}=E_{\infty}^{0, n-d+1}, E_{3}^{1, n-d+1}=E_{\infty}^{1, n-d+1}, E_{3}^{2, n-d}=E_{\infty}^{2, n-d}$, $E_{4}^{0, n-d+2}=E_{\infty}^{0, n-d+2}$ and $E_{4}^{3, n-d}=E_{\infty}^{3, n-d}$.

\section{LineAR STRANDS OF Minimal FREE RESOLUTIONS OF $\mathbb{Z}$-GRADED IDEALS}

Throughout this section we will consider $\mathbb{Z}$-graded ideals $I$ in the polynomial ring $R=\mathbb{k}\left[x_{1}, \ldots, x_{n}\right]$, in particular $I$ is not necessarily a monomial ideal. For simplicity, we will assume that $I \neq 0$. The minimal $\mathbb{Z}$-graded free resolution of $I$ is an exact sequence of free $\mathbb{Z}$-graded modules:

$$
L_{\bullet}(I): \quad 0 \longrightarrow L_{n} \stackrel{d_{n}}{\longrightarrow} \cdots \longrightarrow L_{1} \stackrel{d_{1}}{\longrightarrow} L_{0} \longrightarrow I \longrightarrow 0,
$$

where the $i$-th term is of the form

$$
L_{i}=\bigoplus_{j \in \mathbb{Z}} R(-j)^{\beta_{i, j}(I)}
$$

and the matrices of the morphisms $d_{i}: L_{i} \longrightarrow L_{i-1}$ do not contain invertible elements. The Betti numbers of $I$ are the invariants $\beta_{i, j}(I)$. Notice that $L_{i} \cong R^{\beta_{i}(I)}$ as underlying $R$-modules where, for each $i$, we set $\beta_{i}(I):=\sum_{j \in \mathbb{Z}} \beta_{i, j}(I)$. Hence, (3.1) implies that

$$
\sum_{0 \leq i \leq n}(-1)^{i} \beta_{i}(I)=\operatorname{rank}_{R}(I)=1
$$

Given $r \in \mathbb{N}$, we also consider the $r$-linear strand of $L_{\bullet}(I)$ :

$$
\mathbb{L}_{\bullet}^{<r>}(I): \quad 0 \longrightarrow L_{n}^{<r>} \stackrel{d_{n}^{<r>}}{\longrightarrow} \cdots \longrightarrow L_{1}^{<r>} \stackrel{d_{1}^{<r>}}{\longrightarrow} L_{0}^{<r>} \longrightarrow 0,
$$

where

$$
L_{i}^{<r>}=R(-i-r)^{\beta_{i, i+r}(I)},
$$

and the differential $d_{i}^{<r>}: L_{i}^{<r>} \longrightarrow L_{i-1}^{<r>}$ is the corresponding component of $d_{i}$.

Remark 3.1. Sometimes we will also consider the minimal $\mathbb{Z}$-graded free resolution $L \bullet(R / I)$ of the quotient ring $R / I$ :

$$
L_{\bullet}(R / I): \quad 0 \longrightarrow L_{n} \stackrel{d_{n}}{\longrightarrow} \cdots \longrightarrow L_{1} \stackrel{d_{1}}{\longrightarrow} L_{0}=R \longrightarrow R / I \longrightarrow 0,
$$

Its truncation at the first term $L_{\geq 1}(R / I)$ gives a minimal free resolution $L_{\bullet}(I)$ of $I$. For $r \geq 2, \mathbb{L}_{\bullet}^{<r>}(I)$ is isomorphic to the $(r-1)$-linear strand $\mathbb{L}_{\bullet}^{<r-1>}(R / I)$ up to translation. However, this is not true for $r=1$, since $\mathbb{L}_{\bullet}^{<0>}(R / I)$ starts from the 0 -th term $R$, which is irrelevant to $\mathbb{L}_{\bullet}^{<1>}(I)$. 
To the minimal $\mathbb{Z}$-graded free resolution of $I$ we may associate a set of invariants that measure the acyclicity of the linear strands as follows: Let $\mathbb{K}$ denote the field of fractions $Q(R)$ of $R$, and set

$$
\nu_{i, j}(I):=\operatorname{dim}_{\mathbb{K}}\left[H_{i}\left(\mathbb{L}_{\bullet}^{<j-i>}(I) \otimes_{R} \mathbb{K}\right)\right] .
$$

Since the complex $\mathbb{L}_{\bullet}^{<r>}(I) \otimes_{R} \mathbb{K}$ is of the form

$$
0 \longrightarrow \mathbb{K}^{\beta_{n, n+r}(I)} \stackrel{\partial_{n}^{<r>}}{\longrightarrow} \cdots \longrightarrow \mathbb{K}^{\beta_{1,1+r}(I)} \stackrel{\partial_{1}^{<r>}}{\longrightarrow} \mathbb{K}^{\beta_{0, r}(I)} \longrightarrow 0,
$$

we have $\nu_{i, j}(I) \leq \beta_{i, j}(I)$ for all $i, j$ (if $i>j$ then $\nu_{i, j}(I)=\beta_{i, j}(I)=0$ ), and

$$
\sum_{i=0}^{n}(-1)^{i} \nu_{i, i+r}(I)=\sum_{i=0}^{n}(-1)^{i} \beta_{i, i+r}(I)
$$

for each $r$. If we mimic the construction of the Betti table, we may also consider the $\nu$-table of $I$

\begin{tabular}{c|rrrr}
$\nu_{i, i+r}(I)$ & 0 & 1 & 2 & $\cdots$ \\
\hline 0 & $\nu_{0,0}(I)$ & $\nu_{1,1}(I)$ & $\nu_{2,2}(I)$ & $\cdots$ \\
1 & $\nu_{0,1}(I)$ & $\nu_{1,2}(I)$ & $\nu_{2,3}(I)$ & $\cdots$ \\
$\vdots$ & $\vdots$ & $\vdots$ & $\vdots$ &
\end{tabular}

Next we consider some basic properties of $\nu$-numbers. It turns out that they satisfy analogous properties to those of Lyubeznik numbers. For instance, these invariants satisfy the following Euler characteristic formula.

Lemma 3.2. For a $\mathbb{Z}$-graded ideal $I$, we have

$$
\sum_{i, j \in \mathbb{N}}(-1)^{i} \nu_{i, j}(I)=1
$$

Proof. The assertion follows from the computation below.

$$
\begin{aligned}
& \sum_{i, j \in \mathbb{N}}(-1)^{i} \nu_{i, j}(I) \\
= & \sum_{i, r \in \mathbb{N}}(-1)^{i} \nu_{i, i+r}(I) \\
= & \sum_{r \in \mathbb{N}} \sum_{0 \leq i \leq n}(-1)^{i} \nu_{i, i+r}(I) \\
= & \sum_{r \in \mathbb{N}} \sum_{0 \leq i \leq n}(-1)^{i} \beta_{i, i+r}(I) \\
= & \sum_{0 \leq i \leq n} \sum_{r \in \mathbb{N}}(-1)^{i} \beta_{i, i+r}(I) \\
= & \sum_{0 \leq i \leq n}(-1)^{i} \beta_{i}(I) \\
= & 1
\end{aligned}
$$


We can also single out a particular non-vanishing $\nu$-number. For each $i \in \mathbb{N}$, let $I_{<i>}$ denote the ideal generated by the homogeneous component $I_{i}=\{f \in I \mid \operatorname{deg}(f)=i\} \cup\{0\}$. Then we have:

Lemma 3.3. If $I$ is a $\mathbb{Z}$-graded ideal with $l:=\min \left\{i \mid I_{i} \neq 0\right\}$, then we have $\nu_{0, l}(I) \neq 0$.

Proof. It is easy to see that there is a surjection $H_{0}\left(\mathbb{L}_{\bullet}^{<l>}(I)\right) \rightarrow I_{<l>}$. Since $\operatorname{dim}_{R} I_{<l>}=n$, we have $H_{0}\left(\mathbb{L}_{\bullet}^{<l>}(I) \otimes_{R} \mathbb{K}\right) \cong H_{0}\left(\mathbb{L}_{\bullet}^{<l>}(I)\right) \otimes_{R} \mathbb{K} \neq 0$.

This fact allows us to consider the following notion:

Definition 3.4. Let $I$ be a $\mathbb{Z}$-graded ideal and set $l:=\min \left\{i \mid I_{i} \neq 0\right\}$. We say that $I$ has trivial $\nu$-table, if $\nu_{0, l}(I)=1$ and the rest of these invariants vanish.

3.1. Componentwise linear ideals. It might be an interesting problem to find necessary and/or sufficient conditions for a $\mathbb{Z}$-graded ideal to have a trivial $\nu$-table. In this direction we have the following relation to the notion of componentwise linear ideals.

Definition 3.5 (Herzog and Hibi, [8]). We say a $\mathbb{Z}$-graded ideal $I$ is componentwise linear if $I_{<r>}$ has a linear resolution for all $r \in \mathbb{N}$, i.e., $\beta_{i, j}\left(I_{<r>}\right)=0$ unless $j=i+r$.

Römer $([20])$ and the second author ([25, Theorem 4.1]) independently showed that $I$ is componentwise linear if and only if $H_{i}\left(\mathbb{L}_{\bullet}^{<r>}(I)\right)=0$ for all $r$ and all $i \geq 1$.

Proposition 3.6. A componentwise linear ideal I has a trivial $\nu$-table.

Proof. Since $I$ is componentwise linear, we have $H_{i}\left(\mathbb{L}_{\bullet}^{<r>}(I)\right)=0$ for all $r$ and all $i \geq 1$, and hence $\nu_{i, j}(I)=0$ for all $j$ and all $i \geq 1$. Now the assertion follows from Lemmas 3.2 and 3.3 .

The converse of the above proposition is not true. For example, in Corollary 3.13 below, we will show that if $I_{1} \neq 0$ then it has trivial $\nu$-table. However, there is no relation between being componentwise linear and $I_{1} \neq 0$.

3.2. Consecutiveness of nontrivial columns of the $\nu$-tables. For a $\mathbb{Z}$-graded ideal $I \subseteq R$ and $i \in \mathbb{N}$, set

$$
\nu_{i}(I)=\sum_{j \in \mathbb{N}} \nu_{i, j}(I) .
$$

If we denote $\mathbb{L}_{\bullet}(I):=\bigoplus_{r \in \mathbb{N}} \mathbb{L}_{\bullet}^{<r>}(I)$, then

$$
\nu_{i}(I)=\operatorname{dim}_{\mathbb{K}} H_{i}\left(\mathbb{L}_{\bullet}(I) \otimes_{R} \mathbb{K}\right) .
$$

By Lemma 3.2, we have $\sum_{i=0}^{n}(-1)^{i} \nu_{i}(I)=1$. We say $\nu_{i}(I)$ is non-trivial, if

$$
\nu_{i}(I) \geq \begin{cases}2 & \text { if } i=0 \\ 1 & \text { if } i \geq 1\end{cases}
$$


Clearly, the $\nu$-table of $I$ is non-trivial if and only if $\nu_{i}(I)$ is non-trivial for some $i$. If $n \geq 1$, we have proj. $\operatorname{dim}_{R} I \leq n-1$, and hence $\nu_{n}(I)=0$. In particular, $\nu_{n}(I)$ is always trivial.

The main result of this subsection is the following:

Theorem 3.7. Let $I$ be a $\mathbb{Z}$-graded ideal of $R$. Then;

- If $\nu_{j}(I)$ is non-trivial for $1 \leq j \leq n-1$, then either $\nu_{j-1}(I)$ or $\nu_{j+1}(I)$ is nontrivial.

- If $\nu_{0}(I)$ is non-trivial, then so is $\nu_{1}(I)$.

In order to prove the theorem, we will reconstruct $\mathbb{L}_{\bullet}(I)$ using a spectral sequence. Let $L_{\bullet}(I)$ be the minimal free resolution of $I$ as before. Consider the $\mathfrak{m}$-adic filtration $L_{\bullet}(I)=$ $F_{0} L_{\bullet} \supset F_{1} L_{\bullet} \supset \cdots$ of $L_{\bullet}(I)$, where $F_{i} L_{\bullet}$ is a subcomplex whose component of homological degree $j$ is $\mathfrak{m}^{i} L_{j}$. For any given $R$-module $M$, we regard $\operatorname{gr}(M):=\bigoplus_{i \in \mathbb{N}} \mathfrak{m}^{i} M / \mathfrak{m}^{i+1} M$ as an $R$-module via the isomorphism gr $R=\bigoplus_{i \in \mathbb{N}} \mathfrak{m}^{i} / \mathfrak{m}^{i+1} \cong R=\mathbb{k}\left[x_{1}, \ldots, x_{n}\right]$. Since each $L_{j}$ is a free $R$-module,

$$
\bigoplus_{p+q=-j} E_{0}^{p, q}=\left(\bigoplus_{p \geq 0} \mathfrak{m}^{p} L_{j} / \mathfrak{m}^{p+1} L_{j}\right)=\operatorname{gr} L_{j}
$$

is isomorphic to $L_{j}$ (if we identify gr $R$ with $R$ ), while we have to forget the original $\mathbb{Z}$-grading of $L_{j}$. Since $L_{\bullet}(I)$ is a minimal free resolution, $d_{0}^{p, q}: E_{0}^{p, q} \rightarrow E_{0}^{p, q+1}$ is the zero map for all $p, q$, and hence $E_{0}^{p, q}=E_{1}^{p, q}$. It follows that

$$
\mathbb{E}_{j}^{(1)}:=\bigoplus_{p+q=-j} E_{1}^{p, q}=\bigoplus_{p+q=-j} E_{0}^{p, q}
$$

is isomorphic to $L_{j}$ under the identification $R \cong \operatorname{gr} R$. Collecting the maps

$$
d_{1}^{p, q}: E_{1}^{p, q}\left(=\mathfrak{m}^{p} L_{j} / \mathfrak{m}^{p+1} L_{j}\right) \longrightarrow E_{1}^{p+1, q}\left(=\mathfrak{m}^{p+1} L_{j-1} / \mathfrak{m}^{p+2} L_{j-1}\right)
$$

for $p, q$ with $p+q=-j$, we have the $R$-morphism $d_{j}^{(1)}: \mathbb{E}_{j}^{(1)} \rightarrow \mathbb{E}_{j-1}^{(1)}$, and these morphisms make $\mathbb{E}_{\bullet}^{(1)}$ a chain complex of $R$-modules. Under the isomorphism $\mathbb{E}_{j}^{(1)} \cong L_{j}$, $\mathbb{E}_{\bullet}^{(1)}$ is isomorphic to $\mathbb{L}_{\bullet}(I)=\bigoplus_{r \in \mathbb{N}} \mathbb{L}_{\bullet}^{<r>}(I)$. Hence we have

$$
\mathbb{E}_{j}^{(2)}:=\bigoplus_{p+q=-j} E_{2}^{p, q} \cong H_{j}\left(\mathbb{L}_{\bullet}(I)\right)
$$

and $\nu_{j}(I)=\operatorname{dim}_{\mathbb{K}}\left(\mathbb{E}_{j}^{(2)} \otimes_{R} \mathbb{K}\right)$. Collecting the maps $d_{2}^{p, q}: E_{2}^{p, q} \rightarrow E_{2}^{p+2, q-1}$, we have the $R$-morphism

$$
d_{j}^{(2)}: \mathbb{E}_{j}^{(2)}\left(\cong H_{j}\left(\mathbb{L}_{\bullet}(I)\right)\right) \longrightarrow \mathbb{E}_{j-1}^{(2)}\left(\cong H_{j-1}\left(\mathbb{L}_{\bullet}(I)\right) .\right.
$$

Moreover, we have the chain complex

$$
\cdots \longrightarrow \mathbb{E}_{j+1}^{(2)} \stackrel{d_{j+1}^{(2)}}{\longrightarrow} \mathbb{E}_{j}^{(2)} \stackrel{d_{j}^{(2)}}{\longrightarrow} \mathbb{E}_{j-1}^{(2)} \longrightarrow \cdots,
$$

of $R$-modules whose $j$ th homology is isomorphic to $\mathbb{E}_{j}^{(3)}:=\bigoplus_{p+q=-j} E_{3}^{p, q}$. For all $r \geq 4$, $\mathbb{E}_{j}^{(r)}:=\bigoplus_{p+q=-j} E_{r}^{p, q}$ satisfies the same property. 
By the construction of spectral sequences, if

$$
r>\max \left\{k \mid \beta_{j, k}(I) \neq 0\right\}-\min \left\{k \mid \beta_{j-1, k}(I) \neq 0\right\},
$$

then the map $d_{r}^{p, q}: E_{r}^{p, q} \rightarrow E_{r}^{p+r, q-r+1}$ is zero for all $p, q$ with $p+q=-j$, and hence $d_{j}^{(r)}: \mathbb{E}_{j}^{(r)} \rightarrow \mathbb{E}_{j-1}^{(r)}$ is zero. It implies that $\mathbb{E}_{j}^{(r)}$ is isomorphic to

$$
\mathbb{E}_{j}^{(\infty)}:=\bigoplus_{p+q=-j} E_{\infty}^{p, q}
$$

for $r \gg 0$.

Proof of Theorem 3.7. We will prove the assertion by contradiction using the spectral sequence introduced above. First, assume that $\nu_{j}(I)$ is non-trivial for some $2 \leq j \leq n-1$, but both $\nu_{j-1}(I)$ and $\nu_{j+1}(I)$ are trivial (the cases $j=0,1$ can be proved using similar arguments, and we will give a few remarks later). Then we have $\mathbb{E}_{j}^{(2)} \otimes_{R} \mathbb{K} \neq 0$ and $\mathbb{E}_{j+1}^{(2)} \otimes_{R} \mathbb{K}=\mathbb{E}_{j-1}^{(2)} \otimes_{R} \mathbb{K}=0$. It follows that $\mathbb{E}_{j}^{(3)} \otimes_{R} \mathbb{K} \neq 0$, since it is the homology of

$$
\mathbb{E}_{j+1}^{(2)} \otimes_{R} \mathbb{K} \longrightarrow \mathbb{E}_{j}^{(2)} \otimes_{R} \mathbb{K} \longrightarrow \mathbb{E}_{j-1}^{(2)} \otimes_{R} \mathbb{K}
$$

Similarly, we have $\mathbb{E}_{j-1}^{(3)} \otimes_{R} \mathbb{K}=\mathbb{E}_{j+1}^{(3)} \otimes_{R} \mathbb{K}=0$. Repeating this argument, we have $\mathbb{E}_{j}^{(r)} \otimes_{R} \mathbb{K} \neq 0$ for all $r \geq 4$. Hence $E_{\infty}^{p, q} \neq 0$ for some $p, q$ with $p+q=-j$. However it contradicts the facts that

$$
E_{r}^{p, q} \Longrightarrow H_{-p-q}\left(L_{\bullet}(I)\right)
$$

and $H_{j}\left(L_{\bullet}(I)\right)=0$ (recall that $j>0$ now).

Next we assume that $\nu_{1}(I)$ is non-trivial, but $\nu_{0}(I)$ and $\nu_{2}(I)$ are trivial, that is,

$$
\mathbb{E}_{1}^{(2)} \otimes_{R} \mathbb{K} \neq 0, \quad \mathbb{E}_{0}^{(2)} \otimes_{R} \mathbb{K} \cong \mathbb{K} \quad \text { and } \quad \mathbb{E}_{2}^{(2)} \otimes_{R} \mathbb{K}=0 .
$$

As we have seen above, we must have $\mathbb{E}_{1}^{(r)}=0$ for $r \gg 0$. Since $\mathbb{E}_{2}^{(r)} \otimes_{R} \mathbb{K}=0$ for all $r$ now, if $d_{1}^{(r)} \otimes_{R} \mathbb{K}: \mathbb{E}_{1}^{(r)} \otimes_{R} \mathbb{K} \longrightarrow \mathbb{E}_{0}^{(r)} \otimes_{R} \mathbb{K}$ are the zero maps for all $r$, then $\mathbb{E}_{1}^{(r)} \otimes_{R} \mathbb{K} \cong \mathbb{E}_{1}^{(2)} \otimes_{R} \mathbb{K} \neq 0$ for all $r$, and this is a contradiction. So there is some $r \geq 2$ such that $d_{1}^{(r)} \otimes_{R} \mathbb{K}$ is not zero. If $s$ is the minimum among these $r, d_{1}^{(s)} \otimes_{R} \mathbb{K}$ : $\mathbb{E}_{1}^{(s)} \otimes_{R} \mathbb{K} \longrightarrow\left(\mathbb{E}_{0}^{(s)} \otimes_{R} \mathbb{K}\right) \cong \mathbb{K}$ is surjective. Hence $\mathbb{E}_{0}^{(r)} \otimes_{R} \mathbb{K}=0$ for all $r>s$, and $\mathbb{E}_{0}^{(\infty)} \otimes_{R} \mathbb{K}=0$. However, since $\mathbb{E}_{0}^{(\infty)} \cong \operatorname{gr}\left(H_{0}\left(L_{\bullet}(I)\right) \cong \operatorname{gr}(I)\right.$ and $\operatorname{dim}_{R} I=n$, we have $\operatorname{dim}_{R}(\operatorname{gr}(I))=n$ and hence $\mathbb{E}_{0}^{(\infty)} \otimes_{R} \mathbb{K} \neq 0$. This is a contradiction. The case when $\nu_{0}(I)$ is non-trivial can be proved in a similar way.

3.3. Thom-Sebastiani type formulae. Let $I, J$ be $\mathbb{Z}$-graded ideals in two disjoint sets of variables, say $I \subseteq R=\mathbb{k}\left[x_{1}, \ldots, x_{m}\right]$ and $J \subseteq S=\mathbb{k}\left[y_{1}, \ldots, y_{n}\right]$. The aim of this subsection is to describe the $\nu$-numbers of $I T+J T$, where $T=R \otimes_{\mathbb{k}} S=\mathbb{k}\left[x_{1}, \ldots, x_{m}, y_{1}, \ldots, y_{n}\right]$, in terms of those of $I$ and $J$ respectively. When we just consider Betti numbers we have the following results due to Jacques-Katzman [11. 
Proposition 3.8 (c.f. [11, Lemma 2.1]). Let $L_{\bullet}(R / I)$ and $L_{\bullet}(S / J)$ be minimal graded free resolutions of $R / I$ and $S / J$ respectively. Then,

$$
\left(L \bullet(R / I) \otimes_{R} T\right) \otimes_{T}\left(L \bullet(S / J) \otimes_{S} T\right)
$$

is a minimal graded free resolution of $T / I T+J T$.

Hence, Betti numbers satisfy the following relation:

Corollary 3.9 (c.f. [11, Corollary 2.2]). The Betti numbers of T/IT $+J T$ have the following form:

$$
\beta_{i, j}(T / I T+J T)=\sum_{\substack{k+k^{\prime}=i \\ l+l^{\prime}=j}} \beta_{k, l}(T / I T) \beta_{k^{\prime}, l^{\prime}}(T / J T)
$$

Hence we have

$$
\beta_{i, j}(I T+J T)=\beta_{i, j}(I T)+\beta_{i, j}(J T)+\sum_{\substack{k+k^{\prime}=i-1 \\ l+l^{\prime}=j}} \beta_{k, l}(I T) \beta_{k^{\prime}, l^{\prime}}(J T) .
$$

Our aim is to extend the result in [11] to the case of $\nu$-numbers. To such purpose it will be more convenient to consider separately the case of ideals with degree one elements. Thus, let $I \subseteq R$ be any $\mathbb{Z}$-graded ideal and assume for simplicity that $J$ is principally generated by an element of degree one, e.g. $J=(y) \subseteq S$.

Lemma 3.10. Let $I \subseteq R=\mathbb{k}\left[x_{1}, \ldots, x_{m}\right]$ and $J=(y) \subseteq S=\mathbb{k}[y]$ be $\mathbb{Z}$-graded ideals and set $T=R \otimes_{\mathbb{k}} S=\mathbb{k}\left[x_{1}, \ldots, x_{m}, y\right]$. For $r \geq 2$, the $r$-linear strand $\mathbb{L}_{\bullet}^{<r>}(I T+J T)$ is the mapping cone of the chain map

$$
\times y:\left(\mathbb{L}_{\bullet}^{<r>}(I T)\right)(-1) \rightarrow \mathbb{L}_{\bullet}^{<r>}(I T) .
$$

Proof. It is easy to see that a minimal $T$-free resolution $L_{\bullet}(T / I T+J T)$ of $T / I T+J T$ is given by the mapping cone of the chain map $\times y: L_{\bullet}(T / I T)(-1) \rightarrow L_{\bullet}(T / I T)$, where $L_{\bullet}(T / I T)$ is a minimal $T$-free resolution of $T / I T$. Since the operation of taking $r$-linear strand commutes with the operation of taking the mapping cone, we are done.

The general case is more involved. Assume now that $I \subseteq R=\mathbb{k}\left[x_{1}, \ldots, x_{m}\right]$ and $J \subseteq$ $S=\mathbb{k}\left[y_{1}, \ldots, y_{n}\right]$ are $\mathbb{Z}$-graded ideals such that $I_{1}=0$ and $J_{1}=0$. Let $L_{\bullet}(I)$ be a minimal graded $R$-free resolution of $I$ and $L_{\bullet}(J)$ a minimal graded $S$-free resolution of $J$ and consider their extensions $L_{\bullet}(I T)$ and $L_{\bullet}(J T)$ to $T=R \otimes_{\mathbb{k}} S=\mathbb{k}\left[x_{1}, \ldots, x_{m}, y_{1}, \ldots, y_{n}\right]$.

Lemma 3.11. Under the previous assumptions, the $r$-linear strand $\mathbb{L}_{\bullet}^{<r>}(I T+J T)$ is

$$
\mathbb{L}_{\bullet}^{<r>}(I T+J T)=\mathbb{L}_{\bullet}^{<r>}(I T) \oplus \mathbb{L}_{\bullet}^{<r>}(J T) \oplus\left(\bigoplus_{a+b=r+1}\left(\mathbb{L}_{\bullet}^{<a>}(I T) \otimes_{T} \mathbb{L}_{\bullet}^{<b>}(J T)\right)[-1]\right) .
$$

Here, for a chain complex $C_{\bullet}, C_{\bullet}[-1]$ denotes the translated complex whose component of homological degree $j$ is $C_{j-1}$. 
Proof. Consider the minimal $\mathbb{Z}$-graded free resolutions of $R / I$ and $S / J$ respectively

$$
\begin{aligned}
& L_{\bullet}(R / I): \quad 0 \longrightarrow L_{m} \stackrel{d_{m}}{\longrightarrow} \cdots \longrightarrow L_{1} \stackrel{d_{1}}{\longrightarrow} L_{0} \longrightarrow R / I \longrightarrow 0 \\
& L_{\bullet}^{\prime}(S / J): \quad 0 \longrightarrow L_{n}^{\prime} \stackrel{d_{n}^{\prime}}{\longrightarrow} \cdots \longrightarrow L_{1}^{\prime} \stackrel{d_{1}^{\prime}}{\longrightarrow} L_{0}^{\prime} \longrightarrow S / J \longrightarrow 0,
\end{aligned}
$$

where $L_{0}=R$ and $L_{0}^{\prime}=S$. According to Proposition 3.8, the minimal $\mathbb{Z}$-graded free resolution $L_{\bullet}(T / I T+J T)$ has the form 2 :

$$
\begin{aligned}
& L_{2} \otimes L_{0}^{\prime} \\
& \cdots \longrightarrow L_{1} \otimes L_{1}^{\prime} \stackrel{\partial_{2}}{\longrightarrow} \stackrel{L_{1} \otimes L_{0}^{\prime}}{\oplus} \stackrel{\partial_{1}}{\longrightarrow} L_{0} \otimes L_{0}^{\prime} \longrightarrow T / I T+J T \longrightarrow 0 \\
& \oplus \quad L_{0} \otimes L_{1}^{\prime} \\
& L_{0} \otimes L_{2}^{\prime}
\end{aligned}
$$

where, for any given $x_{i} \otimes y_{p-i} \in L_{i} \otimes L_{p-i}^{\prime}$, we have

$$
\partial_{p}\left(x_{i} \otimes y_{p-i}\right)=d_{i}\left(x_{i}\right) \otimes y_{p-i}+(-1)^{i} x_{i} \otimes d_{p-i}^{\prime}\left(y_{p-i}\right) \in\left(L_{i-1} \otimes L_{p-i}^{\prime}\right) \oplus\left(L_{i} \otimes L_{p-i-1}^{\prime}\right) .
$$

To describe the $r$-linear strand $\mathbb{L}_{\bullet}^{<r>}(I T+J T)$ of the ideal $I T+J T$ we must consider the truncation at the first term of the above resolution and take a close look at the free modules and the components of the corresponding differentials. Recall that $L_{\bullet}^{<r-1>}(R / I)$ corresponds to $L_{\bullet}^{<r>}(I)$ for all $r \geq 2$. It is easy to see that both

$$
\mathbb{L}_{\bullet}^{<r>}(I T): 0 \longrightarrow L_{m}^{<r-1>} \otimes L_{0}^{\prime} \longrightarrow \cdots \longrightarrow L_{2}^{<r-1>} \otimes L_{0}^{\prime} \longrightarrow L_{1}^{<r-1>} \otimes L_{0}^{\prime} \longrightarrow 0
$$

and

$$
\mathbb{L}_{\bullet}^{<r>}(J T): 0 \longrightarrow L_{0} \otimes L_{n}^{\prime<r-1>} \longrightarrow \cdots \longrightarrow L_{0} \otimes L_{2}^{\prime<r-1>} \longrightarrow L_{0} \otimes L_{1}^{\prime<r-1>} \longrightarrow 0
$$

are subcomplexes of $\mathbb{L}_{\bullet}^{<r>}(I T+J T)$. Moreover, $\mathbb{L}_{\bullet}^{<r>}(I T)$ and $\mathbb{L}_{\bullet}^{<r>}(J T)$ are direct summands of $\mathbb{L}_{\bullet}^{<r>}(I T+J T)$. In fact, since $I_{1}=J_{1}=0$, the linear parts of the maps $L_{i} \otimes L_{1}^{\prime} \rightarrow L_{i} \otimes L_{0}^{\prime}$ and $L_{1} \otimes L_{j}^{\prime} \rightarrow L_{0} \otimes L_{j}^{\prime}$ vanish.

In order to obtain the remaining components of $\mathbb{L}_{\bullet}^{<r>}(I T+J T)$ we must consider the $r$-linear strand of

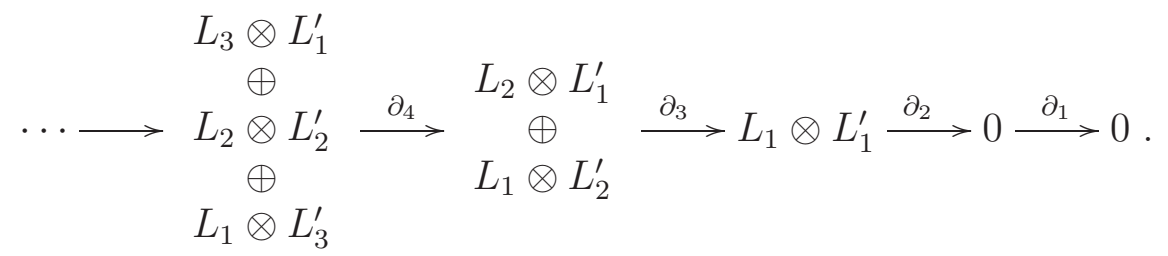

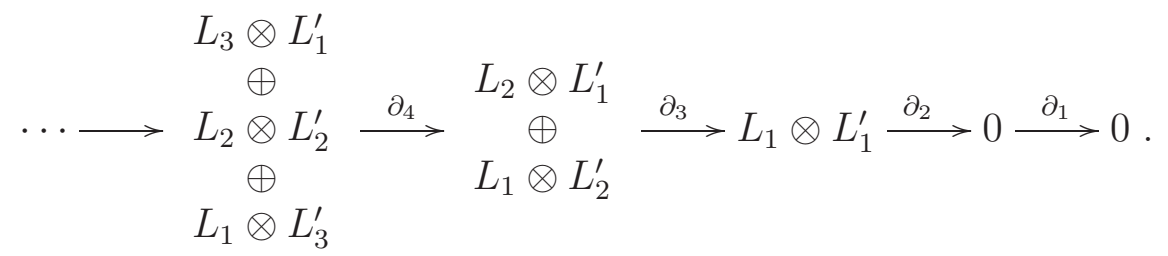

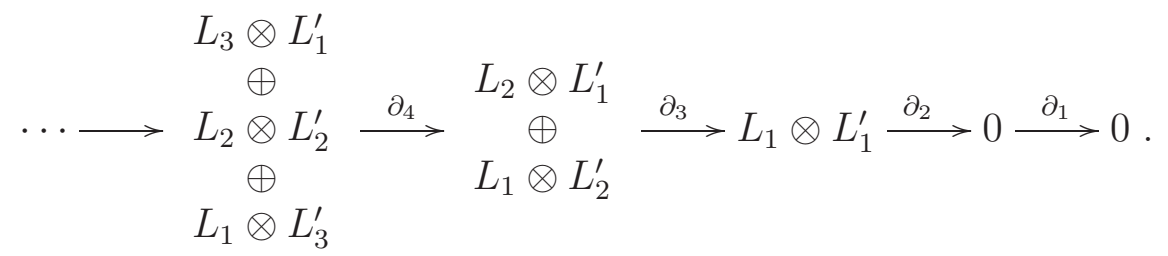

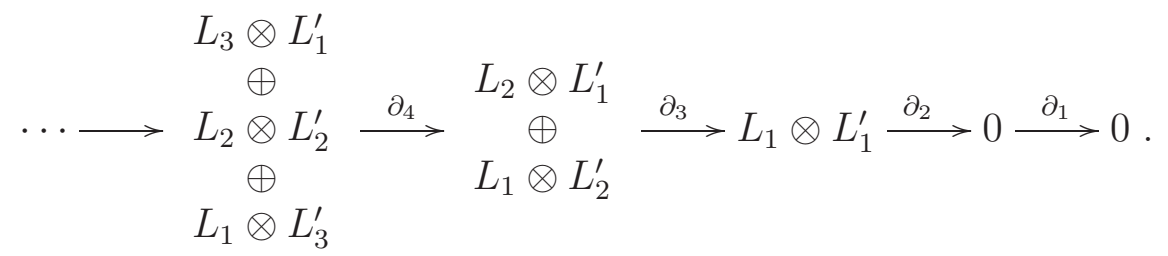

This complex starts at the second term (i.e., the term of homological degree 1), and the first term of the $r$-linear strand is $\bigoplus_{a+b=r+1} \mathbb{L}_{0}^{<a>}(I T) \otimes_{T} \mathbb{L}_{0}^{<b>}(J T)$. If we take a close look

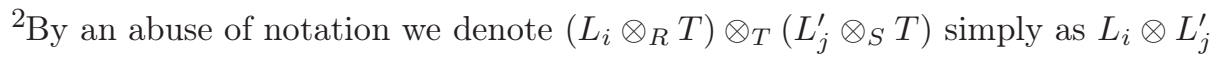


at the free summands of these components and its differentials we obtain the following description

$$
\bigoplus_{a+b=r+1}\left(\mathbb{L}_{\bullet}^{<a>}(I T) \otimes_{T} \mathbb{L}_{\bullet}^{<b>}(J T)\right)[-1]
$$

So we are done.

The main result of this subsection is the following:

Proposition 3.12. The $\nu$-numbers of $I T+J T$ have the following form:

i) If $I_{1} \neq 0$ or $J_{1} \neq 0$ then IT + JT has trivial $\nu$-table.

ii) If $I_{1}=0$ and $J_{1}=0$ then we have:

$$
\nu_{i, j}(I T+J T)=\nu_{i, j}(I T)+\nu_{i, j}(J T)+\sum_{\substack{k+k^{\prime}=i-1 \\ l+l^{\prime}=j}} \nu_{k, l}(I T) \nu_{k^{\prime}, l^{\prime}}(J T) .
$$

Proof. i) If $J_{1} \neq 0$, we may assume that $y_{n} \in J$ without loss of generality. Now we have $J=\left(f_{1}, \ldots, f_{r}, y_{n}\right)$, where $f_{1} \ldots, f_{r}$ are homogeneous polynomials in $\mathbb{k}\left[y_{1}, \ldots y_{n-1}\right]$. Set $R^{\prime}:=\mathbb{k}\left[x_{1}, \ldots, x_{m}, y_{1}, \ldots, y_{n-1}\right], S^{\prime}=\mathbb{k}\left[y_{n}\right]$, and let $I^{\prime}=I R^{\prime}+\left(f_{1}, \ldots, f_{r}\right)$ be an ideal in $R^{\prime}$ (note that $f_{1} \ldots, f_{r}$ are elements in $R^{\prime}$ ), and $J^{\prime}=\left(y_{n}\right)$ an ideal in $S^{\prime}$. Then we have $T=R \otimes_{\mathbb{k}} S=R^{\prime} \otimes_{\mathbb{k}} S^{\prime}$, and $I T+J T=I^{\prime} T+J^{\prime} T$. This means that we may assume that $J=(y) \subseteq S=\mathbb{k}[y]$ from the beginning. For $r \geq 2$, the $r$-linear strand $\mathbb{L}_{\bullet}^{<r>}(I T+J T)$ is given by the mapping cone of the chain map $\times y:\left(\mathbb{L}_{\bullet}^{<r>}(I T)\right)(-1) \rightarrow \mathbb{L}_{\bullet}^{<r>}(I T)$ by Lemma 3.10. Hence $\mathbb{L}_{\bullet}^{<r>}(I T+J T) \otimes_{T} \mathbb{K}$ is given by the mapping cone of the chain map

$$
\times y: \mathbb{L}_{\bullet}^{<r>}(I T) \otimes_{T} \mathbb{K} \longrightarrow \mathbb{L}_{\bullet}^{<r>}(I T) \otimes_{T} \mathbb{K},
$$

where $\mathbb{K}$ is the field of fractions of $T$. Clearly, this is the identity map, and its mapping cone is exact. It means that $H_{i}\left(\mathbb{L}_{\bullet}^{<r>}(I T+J T) \otimes_{T} \mathbb{K}\right)=0$ for all $r \geq 2$ and all $i$.

On the other hand, $(I T+J T)_{<1>}$ is a complete intersection ideal generated by degree 1 elements, and hence we have $\operatorname{dim}_{\mathbb{K}} H_{i}\left(\mathbb{L}_{\bullet}^{<1>}(I) \otimes_{T} \mathbb{K}\right)=\delta_{0, i}$. Summing up, we see that $I T+J T$ has trivial $\nu$-table.

ii) Follows immediately from Lemma 3.11 .

The following is just a rephrasing of part i) of the previous result.

Corollary 3.13. Let $I \subseteq R$ be a $\mathbb{Z}$-graded ideal with $I_{1} \neq 0$, then $I$ has trivial $\nu$-table.

The following is another corollary of Proposition 3.12 .

Corollary 3.14. With the same notation as in Proposition [3.12, if $I_{1}=J_{1}=0$, then $I T+J T$ always has non-trivial $\nu$-table.

Proof. Set $l:=\min \left\{i \mid I_{i} \neq 0\right\}$ and $l^{\prime}:=\min \left\{i \mid J_{i} \neq 0\right\}$. Then we have $\nu_{1, l+l^{\prime}}(I T+J T) \geq$ $\nu_{0, l}(I T) \nu_{0, l^{\prime}}(J T)>0$ by Proposition 3.12 ii). 


\section{LYUBEZNIK NUMBERS VS $\nu$-NUMBERS FOR MONOMIAL IDEALS}

In [26], the second author showed that, via Alexander duality, the study of local cohomology modules with supports in monomial ideals can be "translated" into the study of the minimal free resolutions of squarefree monomial ideals. This fact was later refined by A. Vahidi and the first author in [1] in order to study Lyubeznik numbers of squarefree monomial ideals in terms of the linear strands of its Alexander dual ideals. The aim of this section is to go further in this direction.

In the sequel we will only consider monomial ideals in the polynomial ring $R=$ $\mathbb{k}\left[x_{1}, \ldots, x_{n}\right]$ and $\mathfrak{m}=\left(x_{1}, \ldots, x_{n}\right)$ will denote the graded maximal ideal. Recall that Lyubeznik numbers are well define in this non-local setting since they are invariant with respect to completion so we consider $\lambda_{p, i}(R / I)=\lambda_{p, i}(\widehat{R} / I \widehat{R})$ where $\widehat{R}=\mathbb{k} \llbracket x_{1}, \ldots, x_{n} \rrbracket$. For a vector $\mathbf{a}=\left(a_{1}, \ldots, a_{n}\right) \in \mathbb{N}^{n}$, set $\operatorname{supp}(\mathbf{a}):=\left\{i \mid a_{i} \neq 0\right\} \subseteq\{1, \ldots, n\}$. For each $1 \leq i \leq n$, let $\mathbf{e}_{i} \in \mathbb{Z}^{n}$ be the $i$ th standard vector. The following notion was introduced by the second author, and serves a powerful tool for combinatorial commutative algebra.

Definition 4.1. We say a finitely generated $\mathbb{N}^{n}$-graded $R$-module $M=\bigoplus_{\mathbf{a} \in \mathbb{N}^{n}} M_{\mathbf{a}}$ is squarefree, if the multiplication maps $M_{\mathbf{a}} \ni y \longmapsto x_{i} y \in M_{\mathbf{a}+\mathbf{e}_{i}}$ is bijective for all $\mathbf{a} \in \mathbb{N}^{n}$ and all $i \in \operatorname{supp}(\mathbf{a})$.

The theory of squarefree modules is found in [25, 26, 28, 29]. Here we list some basic properties.

- For a monomial ideal $I$, it is a squarefree $R$-module if and only if $I=\sqrt{I}$ (equivalently, the Stanley-Reisner ideal $I_{\Delta}$ for some $\Delta$ ). The free modules $R$ itself and the $\mathbb{Z}^{n}$-graded canonical module $\omega_{R}=R(-\mathbf{1})$ are squarefree. Here $\mathbf{1}=(1,1, \ldots, 1) \in \mathbb{N}^{n}$. The Stanley-Reisner $\operatorname{ring} R / I_{\Delta}$ is also squarefree.

- Let $M$ be a squarefree $R$-module, and $L$. its $\mathbb{Z}^{n}$-graded minimal free resolution. Then the free module $L_{i}$ and the syzygy module $\operatorname{Syz}_{i}(M)$ are squarefree for each $i$. Moreover, $\operatorname{Ext}_{R}^{i}\left(M, \omega_{R}\right)$ is squarefree for all $i$.

- Let ${ }^{*} \bmod R$ be the category of $\mathbb{Z}^{n}$-graded finitely generated $R$-modules, and $\mathrm{Sq} R$ its full subcategory consisting of squarefree modules. Then $\mathrm{Sq} R$ is an abelian subcategory of $* \bmod R$. We have an exact contravariant functor $\mathbf{A}$ from $\mathrm{Sq} R$ to itself. The construction of $\mathbf{A}$ is found in (for example) [29]. Here we just remark that $\mathbf{A}\left(R / I_{\Delta}\right) \cong I_{\Delta} \vee$, where $\Delta^{\vee}:=\{F \subseteq\{1, \ldots, n\} \mid(\{1, \ldots, n\} \backslash F) \notin \Delta\}$ is the Alexander dual simplicial complex of $\Delta$.

In this framework we have the following description of Lyubeznik numbers.

Theorem 4.2 ([26, Corollary 3.10]). Let $R=\mathbb{k}\left[x_{1}, \ldots, x_{n}\right]$ be a polynomial ring, and $I_{\Delta}$ a squarefree monomial ideal. Then we have

$$
\lambda_{p, i}\left(R / I_{\Delta}\right)=\operatorname{dim}_{\mathbb{k}}\left[\operatorname{Ext}_{R}^{n-p}\left(\operatorname{Ext}_{R}^{n-i}\left(R / I_{\Delta}, \omega_{R}\right), \omega_{R}\right)\right]_{0}<\infty .
$$

For a squarefree $R$-module $M$, the second author defined the cochain complex $\mathbf{D}(M)$ of squarefree $R$-modules satisfying $H^{i}(\mathbf{D}(M)) \cong \operatorname{Ext}_{R}^{n+i}\left(M, \omega_{R}\right)$ for all $i$ (see [29, §3]). By 
[25, Theorem 4.1] or [28, Theorem 3.8], we have the isomorphism

$$
\mathbf{A} \circ \mathbf{D}\left(\operatorname{Ext}_{R}^{n-i}\left(R / I_{\Delta}, \omega_{R}\right)\right) \cong\left(\mathbb{L}_{\bullet}^{<n-i>}\left(I_{\Delta} \vee\right)\right)[-i]
$$

of cochain complexes of $\mathbb{Z}^{n}$-graded $R$-modules 3 . Here, for a cochain complex $C^{\bullet}, C^{\bullet}[-i]$ means the -ith translation of $C^{\bullet}$, more precisely, it is the cochain complex whose component of cohomological degree $j$ is $C^{j-i}$, and we regard a chain complex $C$ • as the cochain complex whose component of cohomological degree $j$ is $C_{-j}$.

The following is a variant of a result given by the first author and A. Vahidi.

Theorem 4.3 (c.f. [1, Corollary 4.2]). Let $I_{\Delta} \subseteq R=\mathbb{k}\left[x_{1}, \ldots, x_{n}\right]$ be a squarefree monomial ideal. Then we have

$$
\lambda_{p, i}\left(R / I_{\Delta}\right)=\nu_{i-p, n-p}\left(I_{\Delta \vee}\right) .
$$

Proof. By (4.1) and the construction of $\mathbf{A}$, we have an isomorphism

$$
\left(\left[\mathbf{D}\left(\operatorname{Ext}_{R}^{n-i}\left(R / I_{\Delta}, \omega_{R}\right)\right]_{0}\right)^{*} \cong\left(\mathbb{L}_{\bullet}^{<n-i>}\left(I_{\Delta} v\right)\right)_{\mathbf{1}}[-i]\right.
$$

of cochain complexes of $\mathbb{k}$-vector spaces. Here $(-)^{*}$ means the $\mathbb{k}$-dual. We also remark that, for a squarefree module $M$, we have

$$
\operatorname{dim}_{\mathbb{k}} M_{\mathbf{1}}=\operatorname{rank}_{R} M=\operatorname{dim}_{\mathbb{K}} M \otimes_{R} \mathbb{K} .
$$

Thus we have the following computation.

$$
\begin{aligned}
\lambda_{p, i}\left(R / I_{\Delta}\right) & \left.=\operatorname{dim}_{\mathbb{k}}\left[\operatorname{Ext}_{R}^{n-p}\left(\operatorname{Ext}_{R}^{n-i}\left(R / I, \omega_{R}\right), \omega_{R}\right)\right)\right]_{0} \\
& =\operatorname{dim}_{\mathbb{k}}\left[H^{-p}\left(\mathbf{D}\left(\operatorname{Ext}_{R}^{n-i}\left(R / I, \omega_{R}\right)\right)\right]_{0}\right. \\
& =\operatorname{dim}_{\mathbb{k}}\left[H_{i-p}\left(\mathbb{L}_{\bullet}^{<n-i>}\left(I_{\Delta} \vee\right)\right]_{\mathbf{1}}\right. \\
& =\operatorname{dim}_{\mathbb{K}} H_{i-p}\left(\mathbb{L}_{\bullet}^{<n-i>}\left(I_{\Delta} \vee\right) \otimes_{R} \mathbb{K}\right. \\
& =\nu_{i-p, n-p}\left(I_{\Delta} \vee\right.
\end{aligned}
$$

As mentioned in Introduction, for a local ring $A$ containing a field, we have

$$
\sum_{0 \leq p, i \leq n}(-1)^{p-i} \lambda_{p, i}(A)=1
$$

In the monomial ideal case, this equation is an immediate consequence of Lemma 3.2 and Theorem 4.3 .

As a special case of Theorem 2.1, the Lyubeznik tables of monomial ideals in $R=$ $\mathbb{k}\left[x_{1}, \ldots, x_{n}\right]$ satisfy the consecutiveness property of nontrivial superdiagonals. However, it also follows from the consecutiveness property of nontrivial columns of the $\nu$-tables (Theorem 3.7) via Theorem 4.3. In this sense, both "consecutiveness theorems" are related.

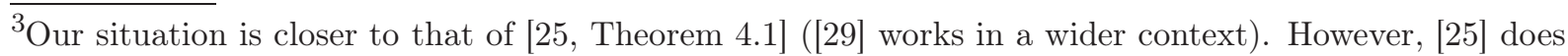
not recognize $\mathbf{D}$ and $\mathbf{A}$ as individual operations, but treats the composition $\mathbf{A} \circ \mathbf{D}$. In fact, $\mathbf{A} \circ \mathbf{D}$ corresponds to the operation $\mathbb{F}_{\bullet}(-)$ of [25] up to translation. 
4.1. Sequentially Cohen-Macaulay rings. Let $M$ be a finitely generated graded module over the polynomial $\operatorname{ring} R=\mathbb{k}\left[x_{1}, \ldots, x_{n}\right]$. We say $M$ is sequentially Cohen-Macaulay if $\operatorname{Ext}_{R}^{n-i}(M, R)$ is either a Cohen-Macaulay module of dimension $i$ or the 0 module for all $i$. The original definition is given by the existence of a certain filtration (see [22, III, Definition 2.9]), however it is equivalent to the above one by [22, III, Theorem 2.11]. The sequentially Cohen-Macaulay property of a finitely generated module over a regular local ring is defined/characterized in the same way.

In [2], the first author showed that the sequentially Cohen-Macaulay property implies the triviality of Lyubeznik tables in positive characteristic as well as in the case of squarefree monomial ideals. Using Proposition 3.6 we can give a new proof/interpretation of this result for the case of monomial ideals.

Proposition 4.4 (c.f. [2, Theorem 3.2]). Let I be a monomial ideal of the polynomial ring $R=\mathbb{k}\left[x_{1}, \ldots, x_{n}\right]$ such that $R / I$ is sequentially Cohen-Macaulay. Then the Lyubeznik table of $R / I$ is trivial.

Proof. By [9, Theorem 2.6], $R / \sqrt{I}$ is sequentially Cohen-Macaulay again. Hence we may assume that $I$ is the Stanley-Reisner ideal $I_{\Delta}$ of a simplicial complex $\Delta$. Herzog and Hibi [8] showed that $R / I_{\Delta}$ is sequentially Cohen-Macaulay if and only if $I_{\Delta} \vee$ is componentwise linear. Now the assertion immediately follows from Proposition 3.6 and Theorem 4.3.

The converse of Proposition 4.4 is not true, that is, even if $R / I$ has trivial Lyubeznik table it need not be sequentially Cohen-Macaulay. For example, if $I$ is the monomial ideal

$$
\left(x_{1}, x_{2}\right) \cap\left(x_{3}, x_{4}\right) \cap\left(x_{1}, x_{5}\right) \cap\left(x_{2}, x_{5}\right) \cap\left(x_{3}, x_{5}\right) \cap\left(x_{4}, x_{5}\right)
$$

in $R=\mathbb{k}\left[x_{1}, \ldots, x_{5}\right]$, then $R / I$ has trivial Lyubeznik table, but this ring is not sequentially Cohen-Macaulay. Since all associated primes of $I$ have the same height, it is the same thing to say $R / I$ is not Cohen-Macaulay. However, $R / I$ does not even satisfy Serre's condition $\left(S_{2}\right)$.

In Proposition 4.5 below, we will see that if a monomial ideal $I$ has height one (i.e., admits a height one associated prime), then the Lyubeznik table of $R / I$ is trivial. Of course, $R / I$ need not be sequentially Cohen-Macaulay in this situation.

4.2. Thom-Sebastiani type formulae. Let $I \subseteq R=\mathbb{k}\left[x_{1}, \ldots, x_{m}\right]$ and $J \subseteq S=$ $\mathbb{k}\left[y_{1}, \ldots, y_{n}\right]$ be squarefree monomial ideals in two disjoint sets of variables. Let $\Delta_{1}$ and $\Delta_{2}$ be the simplicial complexes associated to $I$ and $J$ by the Stanley-Reisner correspondence, i.e. $I=I_{\Delta_{1}}$ and $J=I_{\Delta_{2}}$. Then, the sum $I T+J T=I_{\Delta_{1} * \Delta_{2}}$ corresponds to the simplicial join of both complexes. Let $\Delta_{1}^{\vee}\left(\right.$ resp. $\left.\Delta_{2}^{\vee}\right)$ be the Alexander dual of $\Delta_{1}$ (resp. $\Delta_{2}$ ) as a simplicial complex on $\{1,2, \ldots, m\}$ (resp. $\{1,2, \ldots, n\}$ ). Set $I^{\vee}:=I_{\Delta_{1}^{\vee}} \subseteq R$ and $J^{\vee}:=I_{\Delta_{2}} \subseteq S$. Then it is easy to see that

$$
\mathbf{A}(T / I T) \cong I^{\vee} T, \quad \mathbf{A}(T / J T) \cong J^{\vee} T, \quad \text { and } \quad \mathbf{A}(T / I T \cap J T) \cong I^{\vee} T+J^{\vee} T,
$$

where A denotes the Alexander duality functor of $\mathrm{Sq} T$. 
Proposition 4.5. The Lyubeznik numbers of $T / I T \cap J T$ have the following form:

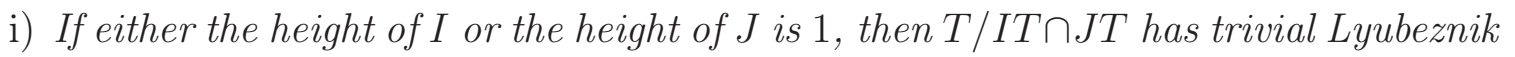
table.

ii) If both the height of I and the height of $J$ are $\geq 2$, then we have:

$$
\begin{aligned}
\lambda_{p, i}(T / I T \cap J T) & =\lambda_{p, i}(T / I T)+\lambda_{p, i}(T / J T)+\sum_{\substack{q+r=p+\operatorname{dim} T \\
j+k=i+\operatorname{dim} T-1}} \lambda_{q, j}(T / I T) \lambda_{r, k}(T / J T) \\
& =\lambda_{p-n, i-n}(R / I)+\lambda_{p-m, i-m}(S / J)+\sum_{\substack{q+r=p \\
j+k=i-1}} \lambda_{q, j}(R / I) \lambda_{r, k}(S / J) .
\end{aligned}
$$

Proof. The assertion easily follows from Proposition 3.12 and Theorem 4.3, but for completeness, we will give a few remarks.

(i) Recall that, for a simplicial complex $\Delta$, the height of $I_{\Delta}$ is 1 if and only if $\left[I_{\Delta \vee}\right]_{1} \neq 0$.

(ii) The last equality follows from the fact that

$$
\lambda_{p, i}(T / I T)=\lambda_{p-n, i-n}(R / I) \quad \text { and } \quad \lambda_{p, i}(T / J T)=\lambda_{p-m, i-m}(S / J),
$$

which can be seen from Theorem 4.3 and the construction of linear strands.

Example 4.6. It is well-know that local cohomology modules as well as free resolutions depend on the characteristic of the base field so Lyubeznik numbers depend on the characteristic as well. The most recurrent example is the Stanley-Reisner ideal associated to a minimal triangulation of $\mathbb{P}_{\mathbb{R}}^{2}$, i.e. the ideal in $R=\mathbb{k}\left[x_{1}, \ldots, x_{6}\right]$ :

$$
I=\left(x_{1} x_{2} x_{3}, x_{1} x_{2} x_{4}, x_{1} x_{3} x_{5}, x_{2} x_{4} x_{5}, x_{3} x_{4} x_{5}, x_{2} x_{3} x_{6}, x_{1} x_{4} x_{6}, x_{3} x_{4} x_{6}, x_{1} x_{5} x_{6}, x_{2} x_{5} x_{6}\right) .
$$

Its Lyubeznik table has been computed in [1, Ex. 4.8]. Namely, in characteristic zero and two respectively, we have:

$$
\Lambda_{\mathbb{Q}}(R / I)=\left(\begin{array}{cccc}
0 & 0 & 0 & 0 \\
& 0 & 0 & 0 \\
& & 0 & 0 \\
& & & 1
\end{array}\right) \quad \Lambda_{\mathbb{Z} / 2 \mathbb{Z}}(R / I)=\left(\begin{array}{cccc}
0 & 0 & 1 & 0 \\
& 0 & 0 & 0 \\
& & 0 & 1 \\
& & & 1
\end{array}\right)
$$

One can slightly modify this example and use Proposition 4.5 to obtain some interesting behavior of Lyubeznik numbers:

- The ideal $J=I \cap\left(x_{7}\right)$ in $R=\mathbb{k}\left[x_{1}, \ldots, x_{7}\right]$ has trivial Lyubeznik table in any characteristic, so we obtain an example where the local cohomology modules depend on the characteristic but Lyubeznik numbers do not.

- The ideal $J=I \cap\left(x_{7}, x_{8}\right) \cap\left(x_{9}, x_{10}\right)$ in $R=\mathbb{k}\left[x_{1}, \ldots, x_{10}\right]$ satisfies

$$
1=\lambda_{6,7}^{\mathbb{Q}}(R / J) \neq \lambda_{6,7}^{\mathbb{Z} / 2 \mathbb{Z}}(R / J)=2
$$

and both Lyubeznik numbers are different from zero. 


\section{LYUBEZNIK TABLE IS A TOPOLOGICAL INVARIANT}

While the other sections treat the case where $R$ is a regular local ring or a polynomial ring, in this section we will work in a slightly different situation. Here the ring $R$ means a normal semigroup ring. When $R$ is simplicial and Gorenstein, the second author proved in [27] that the local cohomology modules $H_{I}^{r}(R)$ have finite Bass numbers for radical monomial ideals $I \subset R$. In fact, without these conditions, Bass numbers are out of control and can be infinite (see [7] for details).

Before going to the main result of this section (Theorem 5.3), we will introduce the setup on which we will work with. For more details we refer to [27].

Let $C \subset \mathbb{Z}^{n}$ be an affine semigroup (i.e., $C$ is a finitely generated additive submonoid of $\left.\mathbb{Z}^{n}\right)$, and $R:=\mathbb{k}\left[\mathbf{x}^{\mathbf{c}} \mid \mathbf{c} \in C\right] \subset \mathbb{k}\left[x_{1}^{ \pm 1}, \ldots, x_{n}^{ \pm 1}\right]$ the semigroup ring of $C$ over $\mathbb{k}$. Here $\mathbf{x}^{\mathbf{c}}$ denotes the monomial $\prod_{i=1}^{n} x_{i}^{c_{i}}$ for $\mathbf{c}=\left(c_{1}, \ldots, c_{n}\right) \in C$. Regarding $C$ as a subset of $\mathbb{R}^{n}=\mathbb{R} \otimes_{\mathbb{Z}} \mathbb{Z}^{n}$, let $P:=\mathbb{R}_{\geq 0} C \subset \mathbb{R}^{n}$ be the polyhedral cone spanned by $C$. We always assume that $\mathbb{Z} C=\mathbb{Z}^{n}, \mathbb{Z}^{n} \cap P=C$ and $C \cap(-C)=\{0\}$. Thus $R$ is a normal Cohen-Macaulay integral domain of dimension $n$ with the graded maximal ideal $\mathfrak{m}:=\left(\mathbf{x}^{\mathbf{c}} \mid 0 \neq \mathbf{c} \in C\right)$. We say $R$ is simplicial, if the cone $P$ is spanned by $n$ vectors in $\mathbb{R}^{n}$. The polynomial ring $\mathbb{k}\left[x_{1}, \ldots, x_{n}\right]$ is a typical example of a simplicial semigroup $\operatorname{ring} \mathbb{k}[C]$ for $C=\mathbb{N}^{n}$. Clearly, $R=\bigoplus_{\mathbf{c} \in C} \mathbb{k} \mathbf{x}^{\mathbf{c}}$ is a $\mathbb{Z}^{n}$-graded ring. We say that a $\mathbb{Z}^{n}$-graded ideal of $R$ is a monomial ideal and we will denote ${ }^{*} \bmod R$ the category of finitely generated $\mathbb{Z}^{n}$-graded $R$-modules and degree preserving $R$-homomorphisms.

Let $L$ be the set of non-empty faces of the polyhedral cone $P$. Note that $\{0\}$ and $P$ itself belong to $L$. Regarding $L$ as a partially ordered set by inclusion, $R$ is simplicial if and only if $L$ is isomorphic to the power set $2^{\{1, \ldots, n\}}$. For $F \in L, \mathfrak{p}_{F}:=\left(\mathbf{x}^{\mathbf{c}} \mid \mathbf{c} \in C \backslash F\right)$ is a prime ideal of $R$. Conversely, any monomial prime ideal is of the form $\mathfrak{p}_{F}$ for some $F \in L$. Note that $R / \mathfrak{p}_{F} \cong \mathbb{k}\left[\mathbf{x}^{\mathbf{c}} \mid \mathbf{c} \in C \cap F\right]$ for $F \in L$. For a point $\mathbf{c} \in C$, we always have a unique face $F \in L$ whose relative interior contains c. Here we denote $s(\mathbf{c})=F$.

The following is a generalization of the notion of squarefree modules (see Definition 4.1) to this setting.

Definition $5.1([27])$. We say a module $M \in{ }^{*} \bmod R$ is squarefree, if it is $C$-graded (i.e., $M_{\mathbf{a}}=0$ for all $\mathbf{a} \notin C$ ), and the multiplication map $M_{\mathbf{a}} \ni y \longmapsto \mathbf{x}^{\mathbf{b}} y \in M_{\mathbf{a}+\mathbf{b}}$ is bijective for all $\mathbf{a}, \mathbf{b} \in C$ with $s(\mathbf{a}+\mathbf{b})=s(\mathbf{a})$.

For a monomial ideal $I, R / I$ is a squarefree $R$-module if and only if $I$ is a radical ideal (i.e., $\sqrt{I}=I$ ). We say that $\Delta \subseteq L$ is an order ideal if $\Delta \ni F \supset F^{\prime} \in L$ implies $F^{\prime} \in \Delta$. If $\Delta$ is an order ideal, then $I_{\Delta}:=\left(\mathbf{x}^{\mathbf{c}} \mid \mathbf{c} \in C, s(\mathbf{c}) \notin \Delta\right) \subseteq R$ is a radical monomial ideal. Conversely, any radical monomial ideal is of the form $I_{\Delta}$ for some $\Delta$. Clearly,

$$
\left[R / I_{\Delta}\right]_{\mathbf{c}} \cong \begin{cases}\mathbb{k} & \text { if } \mathbf{c} \in C \text { and } s(\mathbf{c}) \in \Delta \\ 0 & \text { otherwise }\end{cases}
$$


If $R$ is simplicial, an order ideal $\Delta$ is essentially a simplicial complex on the vertices $1,2, \ldots, n$. If $R$ is the polynomial $\operatorname{ring} \mathbb{k}\left[x_{1}, \ldots, x_{n}\right]$, then $R / I_{\Delta}$ is nothing but the StanleyReisner ring of the simplicial complex $\Delta$.

For each $F \in L$, take some $\mathbf{c}(F) \in C \cap \operatorname{rel}$-int $(F)$ (i.e., $s(\mathbf{c}(F))=F$ ). For a squarefree $R$-module $M$ and $F, G \in L$ with $G \supset F$, [27, Theorem 3.3] gives a $\mathbb{k}$-linear map

$$
\varphi_{G, F}^{M}: M_{\mathbf{c}(F)} \rightarrow M_{\mathbf{c}(G)}
$$

These maps satisfy $\varphi_{F, F}^{M}=\mathrm{Id}$ and $\varphi_{H, G}^{M} \circ \varphi_{G, F}^{M}=\varphi_{H, F}^{M}$ for all $H \supset G \supset F$. We have $M_{\mathbf{c}} \cong M_{\mathbf{c}^{\prime}}$ for $\mathbf{c}, \mathbf{c}^{\prime} \in C$ with $s(\mathbf{c})=s\left(\mathbf{c}^{\prime}\right)$. Under these isomorphisms, the maps $\varphi_{G, F}^{M}$ do not depend on the particular choice of $\mathbf{c}(F)$ 's.

Let $\mathrm{Sq} R$ be the full subcategory of $* \bmod R$ consisting of squarefree modules. As shown in [27], $\mathrm{Sq} R$ is an abelian category with enough injectives. For an indecomposable squarefree module $M$, it is injective in $\operatorname{Sq} R$ if and only if $M \cong R / \mathfrak{p}_{F}$ for some $F \in L$.

Let $\omega_{R}$ be the $\mathbb{Z}^{n}$-graded canonical module of $R$. It is well-known that $\omega_{R}$ is isomorphic to the radical monomial ideal $\left(\mathbf{x}^{\mathbf{c}} \mid \mathbf{c} \in C, s(\mathbf{c})=P\right.$ ). As shown in [27, Proposition 3.7] we have $\operatorname{Ext}_{R}^{i}\left(M, \omega_{R}\right) \in \operatorname{Sq} R$ for $M \in \operatorname{Sq} R$.

5.1. Lyubeznik numbers. Let $R=\mathbb{k}[C]$ be a normal simplicial semigroup ring which is Gorenstein, and $I$ a monomial ideal of $R$. As in the polynomial ring case, we set the Lyubeznik numbers as

$$
\lambda_{p, i}(R / I):=\mu^{p}\left(\mathfrak{m}, H_{I}^{n-i}(R)\right) .
$$

Work of the second author in 27] states that this set of invariants are well defined in this framework. Namely, Theorem 4.2 holds verbatim in this situation.

Theorem 5.2 ([27, Corollary 5.12]). Let $R=\mathbb{k}[C]$ be a normal simplicial semigroup ring which is Gorenstein, and $I_{\Delta}$ a radical monomial ideal. Then we have

$$
\lambda_{p, i}\left(R / I_{\Delta}\right)=\operatorname{dim}_{\mathbb{k}}\left[\operatorname{Ext}_{R}^{n-p}\left(\operatorname{Ext}_{R}^{n-i}\left(R / I_{\Delta}, \omega_{R}\right), \omega_{R}\right)\right]_{0}<\infty .
$$

Notice that in this setting we have that whenever we have a multigraded isomorphism $\mathbb{k}[C] / I_{\Delta} \cong \mathbb{k}\left[C^{\prime}\right] / I_{\Delta^{\prime}}$ between quotients of Gorenstein normal simplicial semigroup rings by radical monomial ideals, then the corresponding Lyubeznik numbers coincide. This multigraded framework slightly differs from the original situation for regular local rings stated in [13]. However, as stated in [27, Remark 5.14], if $\Delta \cong \Delta^{\prime}$ as simplicial complexes, then $R / I_{\Delta}$ and $R^{\prime} / I_{\Delta^{\prime}}$ have the same Lyubeznik numbers. In this sense, to study the Lyubeznik numbers of a quotient $R / I_{\Delta}$ of a Gorenstein normal simplicial semigroup ring $R$ by a radical monomial ideal $I_{\Delta}$, we may assume that $R$ is a polynomial ring and $R / I_{\Delta}$ is a Stanley-Reisner ring. In Theorem 5.3, we will prove a stronger result.

It is also worth to point out that several features of Lyubeznik numbers are still true in this setting. In what follows, we assume that $I$ is a monomial ideal of $R$. 
(1) As in the polynomial ring case, we have the Euler characteristic equation,

$$
\sum_{0 \leq p, i \leq d}(-1)^{p-i} \lambda_{p, i}(R / I)=1
$$

Moreover, the statements corresponding to Theorem 2.1 (the consecutiveness of nontrivial lines) still holds. In fact, we may assume that $I$ is a radical ideal, and hence $I=I_{\Delta}$ for some simplicial complex $\Delta$ and then reduce to the case when $R$ is a polynomial ring as in [27, Remark $5.14(\mathrm{~b})]$.

If we assume that $I=\sqrt{I}$, Proposition 4.4 also holds in the present situation. However, we cannot drop this assumption, since we have no idea whether the condition of being sequentially Cohen-Macaulay is preserved after taking radicals. What is known is that if $R / I$ is Cohen-Macaulay then so is $R / \sqrt{I}$ (see [30, Theorem 6.1]). Hence if $R / I$ is Cohen-Macaulay then the Lyubeznik table of $R / I$ is trivial.

(2) For a radical monomial ideal $I_{\Delta}$ with $\operatorname{dim} R / I_{\Delta}=d$, the highest Lyubeznik number

$$
\lambda_{d, d}\left(R / I_{\Delta}\right)=\operatorname{dim}_{\mathbb{k}}\left[\operatorname{Ext}_{R}^{n-d}\left(\operatorname{Ext}_{R}^{n-d}\left(R / I_{\Delta}, \omega_{R}\right), \omega_{R}\right)\right]_{0}
$$

has a simple topological (or combinatorial) meaning. In fact, to study this number we may assume that $R$ is a polynomial ring, and we can use a combinatorial description of

$$
\operatorname{Ext}_{R}^{n-d}\left(\operatorname{Ext}_{R}^{n-d}\left(R / I_{\Delta}, \omega_{R}\right), \omega_{R}\right)
$$

given in [22, P.96]. Roughly speaking, $\lambda_{d, d}\left(R / I_{\Delta}\right)$ is the number of "connected in codimension one components" of $|\Delta|$. (This result holds in a much wider context, see [31].) In particular, if $R / I_{\Delta}$ satisfies Serre's condition $\left(S_{2}\right)$ then $\lambda_{d, d}\left(R / I_{\Delta}\right)=1$, while the converse is not true.

5.2. Lyubeznik table is a topological invariant. Recall that if $R=\mathbb{k}[C]$ is simplicial then an order ideal $\Delta$ of $L$ is essentially a simplicial complex, and hence it has the geometric realization $|\Delta|$. It is natural to ask how Lyubeznik numbers of $R / I_{\Delta}$ depend on $|\Delta|$. The next theorem shows that Lyubeznik numbers are not only an algebraic invariant but also a topological invariant.

Theorem 5.3. Let $R=\mathbb{k}[C]$ be a simplicial normal semigroup ring which is Gorenstein and $I_{\Delta} \subset R$ a radical monomial ideal. Then, $\lambda_{p, i}\left(R / I_{\Delta}\right)$ depends only on the homeomorphism class of $|\Delta|$ and $\operatorname{char}(\mathbb{k})$.

Bearing in mind Theorem 5.2 , it suffices to show that

$$
\operatorname{dim}_{\mathbb{k}}\left[\operatorname{Ext}_{R}^{n-p}\left(\operatorname{Ext}_{R}^{n-i}\left(R / I_{\Delta}, \omega_{R}\right), \omega_{R}\right)\right]_{0}
$$

depends only on the topology of $|\Delta|$ and $\operatorname{char}(\mathbb{k})$. For this statement, the assumption that $R$ is simplicial and Gorenstein is irrelevant (if $R$ is not simplicial, then $\Delta$ is essentially a CW complex). In [19, Theorem 2.10], R. Okazaki and the second author showed that the invariant which is (essentially) equal to

$$
\operatorname{depth}_{R}\left(\operatorname{Ext}_{R}^{n-i}\left(R / I_{\Delta}, \omega_{R}\right)\right)=\min \left\{j \mid \operatorname{Ext}_{R}^{n-j}\left(\operatorname{Ext}_{R}^{n-i}\left(R / I_{\Delta}, \omega_{R}\right), \omega_{R}\right) \neq 0\right\}
$$


depends only on $|\Delta|$ and $\operatorname{char}(\mathbb{k})$ for each $i$. Our proof here uses similar arguments to the aforementioned result. To do so, we have to recall some previous work of the second author in [28].

Recall that $P=\mathbb{R}_{>0} C$ is a polyhedral cone associated with the semigroup $\operatorname{ring} R=$ $\mathbb{k}[C]$. We have a hyperplane $H \subset \mathbb{R}^{n}$ such that $B:=H \cap P$ is an $(n-1)$-polytope (an $(n-1)$-simplex, if $R$ is simplicial). For $F \in L$, set $|F|$ to be the relative interior of the face $F \cap H$ of $B$. We can regard an order ideal $\Delta \subseteq L$ as a CW complex (a simplicial complex, if $R$ is simplicial) whose geometric realization is $|\Delta|:=\bigcup_{F \in \Delta}|F| \subseteq B$.

For $F \in L$,

$$
U_{F}:=\bigcup_{F^{\prime} \in L, F^{\prime} \supset F}\left|F^{\prime}\right|
$$

is an open set of $B$. Note that $\left\{U_{F} \mid\{0\} \neq F \in L\right\}$ is an open covering of $B$. In [28], from $M \in \operatorname{Sq} R$, we constructed a sheaf $M^{+}$on $B$. More precisely, the assignment

$$
\Gamma\left(U_{F}, M^{+}\right)=M_{\mathbf{c}(F)}
$$

for each $F \neq\{0\}$ and the map

$$
\varphi_{F, G}^{M}: \Gamma\left(U_{G}, M^{+}\right)=M_{\mathbf{c}(G)} \longrightarrow M_{\mathbf{c}(F)}=\Gamma\left(U_{F}, M^{+}\right)
$$

for $F, G \neq\{0\}$ with $F \supset G$ (equivalently, $U_{G} \supset U_{F}$ ) defines a sheaf. Note that $M_{0}$ is "irrelevant" to $M^{+}$.

For example, $\left(R / I_{\Delta}\right)^{+} \cong j_{*} \underline{\mathbb{k}}_{|\Delta|}$, where $\underline{\mathbb{k}}_{|\Delta|}$ is the constant sheaf on $|\Delta|$ with coefficients in $\mathbb{k}$, and $j$ is the embedding map $|\Delta| \hookrightarrow B$. Similarly, we have that $\left(\omega_{R}\right)^{+} \cong h_{!} \underline{\mathbb{k}}_{B^{\circ}}$, where $\underline{\mathbb{k}}_{B^{\circ}}$ is the constant sheaf on the relative interior $B^{\circ}$ of $B$, and $h$ is the embedding map $B^{\circ} \hookrightarrow B$. Note that $\left(\omega_{R}\right)^{+}$is the orientation sheaf of $B$ with coefficients in $\mathbb{k}$.

Let $\Delta \subseteq L$ be an order ideal, and set $X:=|\Delta| \subseteq B$. For $M \in \operatorname{Sq} R, M$ is an $R / I_{\Delta^{-}}$ modules (i.e., $\left.\operatorname{ann}(M) \supset I_{\Delta}\right)$ if and only if $\operatorname{Supp}\left(M^{+}\right):=\left\{x \in B \mid\left(M^{+}\right)_{x} \neq 0\right\} \subseteq X$. In this case, we have

$$
H^{i}\left(B ; M^{+}\right) \cong H^{i}\left(X ;\left.M^{+}\right|_{X}\right)
$$

for all $i$. Here $\left.M^{+}\right|_{X}$ is the restriction of the sheaf $M^{+}$to the closed set $X \subseteq B$. Combining this fact with [28, Theorem 3.3], we have the following.

Theorem 5.4 (c.f. [28, Theorem 3.3]). With the above situation, we have

$$
H^{i}\left(X ;\left.M^{+}\right|_{X}\right) \cong\left[H_{\mathfrak{m}}^{i+1}(M)\right]_{0} \text { for all } i \geq 1,
$$

and an exact sequence

$$
0 \longrightarrow\left[H_{\mathfrak{m}}^{0}(M)\right]_{0} \longrightarrow M_{0} \longrightarrow H^{0}\left(X ;\left.M^{+}\right|_{X}\right) \longrightarrow\left[H_{\mathfrak{m}}^{1}(M)\right]_{0} \longrightarrow 0 .
$$

In particular, $\left[H_{\mathfrak{m}}^{i+1}\left(R / I_{\Delta}\right)\right]_{0} \cong \widetilde{H}^{i}(X ; \mathbb{k})$ for all $i \geq 0$, where $\widetilde{H}^{i}(X ; \mathbb{k})$ denotes the ith reduced cohomology of $X$ with coefficients in $\mathbb{k}$.

Recall that $X$ admits Verdier's dualizing complex $\mathcal{D}_{X}^{\bullet}$ with coefficients in $\mathbb{k}$. For example, $\mathcal{D}_{B}^{\bullet}$ is quasi-isomorphic to $\left(\omega_{R}\right)^{+}[n-1]$. The former half of (1) of the next theorem is a restatement of [28, Theorem 4.2], and the rest is that of [30, Lemma 5.11]. 
Theorem 5.5 ([28, Theorem 4.2] and [30, Lemma 5.11]). With the above notation, we have the following:

(1) $\operatorname{Supp}\left(\operatorname{Ext}_{R}^{n-i}\left(M, \omega_{R}\right)^{+}\right) \subseteq X$ and

$$
\left.\operatorname{Ext}_{R}^{n-i}\left(M, \omega_{R}\right)^{+}\right|_{X} \cong \mathcal{E} x t^{1-i}\left(\left.M^{+}\right|_{X}, \mathcal{D}_{X}^{\bullet}\right) .
$$

Moreover, for $i \geq 2$, we have

$$
\left[\operatorname{Ext}_{R}^{n-i}\left(M, \omega_{R}\right)^{+}\right]_{0} \cong \operatorname{Ext}^{1-i}\left(\left.M^{+}\right|_{X}, \mathcal{D}_{X}^{\bullet}\right)
$$

(2) Via the isomorphisms in (1), for $i \geq 2$, the natural map

$$
\operatorname{Ext}^{1-i}\left(\left.M^{+}\right|_{X}, \mathcal{D}_{X}^{\bullet}\right) \longrightarrow \Gamma\left(X ; \mathcal{E} x t^{1-i}\left(\left.M^{+}\right|_{X}, \mathcal{D}_{X}^{\bullet}\right)\right)
$$

coincides with the middle map

$$
\left[\operatorname{Ext}_{R}^{n-i}\left(M, \omega_{R}\right)\right]_{0} \longrightarrow \Gamma\left(X ;\left.\operatorname{Ext}_{R}^{n-i}\left(M, \omega_{R}\right)^{+}\right|_{X}\right)
$$

of the sequence (5.1) for $\operatorname{Ext}_{R}^{n-i}\left(M, \omega_{R}\right) \in \operatorname{Sq} R$.

The proof of Theorem 5.3. We show that the dimension of $\left[\operatorname{Ext}_{R}^{n-p}\left(\operatorname{Ext}_{R}^{n-i}\left(R / I_{\Delta}, \omega_{R}\right), \omega_{R}\right)\right]_{0}$ $\left(\cong\left[H_{\mathfrak{m}}^{p}\left(\operatorname{Ext}_{R}^{n-i}\left(R / I_{\Delta}, \omega_{R}\right)\right)^{*}\right]_{0}\right)$ depends only on $X$ and $\operatorname{char}(\mathbb{k})$. If $p \geq 2$, then we have

$$
\left[H_{\mathfrak{m}}^{p}\left(\operatorname{Ext}_{R}^{n-i}\left(R / I_{\Delta}, \omega_{R}\right)\right)\right]_{0} \cong H^{p-1}\left(X ; \mathcal{E} x t^{1-i}\left(\underline{\mathbb{k}}_{X}, \mathcal{D}_{X}^{\bullet}\right)\right)
$$

by Theorems 5.4 and 5.5 (1). The right side of the equation clearly depends only on $X$ and $\operatorname{char}(\mathbb{k})$ for each $p, i$. Next we consider the case $p=0,1$. By Theorem [5.4, $H_{\mathfrak{m}}^{0}\left(\operatorname{Ext}_{R}^{n-i}\left(R / I_{\Delta}, \omega_{R}\right)\right)$ and $H_{\mathfrak{m}}^{1}\left(\operatorname{Ext}_{R}^{n-i}\left(R / I_{\Delta}, \omega_{R}\right)\right)$ are the kernel and the cokernel of the map

$$
\left[\operatorname{Ext}_{R}^{n-i}\left(R / I_{\Delta}, \omega_{R}\right)\right]_{0} \longrightarrow \Gamma\left(X ;\left.\operatorname{Ext}_{R}^{n-i}\left(R / I_{\Delta}, \omega_{R}\right)^{+}\right|_{X}\right)
$$

respectively. If $i \geq 2$, the above map is equivalent to the natural map

$$
\operatorname{Ext}^{1-i}\left(\underline{\mathbb{k}}_{X}, \mathcal{D}_{X}^{\bullet}\right) \longrightarrow \Gamma\left(X ; \mathcal{E} x t^{1-i}\left(\underline{\mathbb{k}}_{X}, \mathcal{D}_{X}^{\bullet}\right)\right)
$$

by Theorem $5.5(2)$, and the dimensions of its kernel and cokernel are invariants of $X$.

It remains to show the case $\left(p=0,1\right.$ and) $i=0,1$. Clearly, $\operatorname{Ext}_{R}^{n}\left(R / I_{\Delta}, \omega_{R}\right) \neq 0$, if and only if $\operatorname{Ext}_{R}^{n}\left(R / I_{\Delta}, \omega_{R}\right)=\mathbb{k}$, if and only if $I_{\Delta}=\mathfrak{m}$, if and only if $X=\emptyset$. Hence $\lambda_{0,0}\left(R / I_{\Delta}\right) \neq 0$, if and only if $\lambda_{0,0}\left(R / I_{\Delta}\right)=1$, if and only if $X=\emptyset$. On the other hand, it is easy to check out that $\lambda_{1,1}\left(R / I_{\Delta}\right)$ is always "trivial", that is,

$$
\lambda_{1,1}\left(R / I_{\Delta}\right)= \begin{cases}1 & \text { if } \operatorname{dim}\left(R / I_{\Delta}\right)=1 \quad(\text { i.e. }, \operatorname{dim}|\Delta|=0), \\ 0 & \text { otherwise }\end{cases}
$$

(the same is true for the local ring case using the spectral sequence argument as in the proof of Theorem 2.1 or adapting the techniques used in [24]). Hence the remaining case is only $\lambda_{0,1}\left(R / I_{\Delta}\right)$, but the following fact holds.

Claim. If $R=\mathbb{k}[C]$ is a simplicial normal semigroup ring which is Gorenstein, then we have

$$
\lambda_{0,1}\left(R / I_{\Delta}\right)= \begin{cases}c-1 & \text { if } \left.\operatorname{dim}\left(R / I_{\Delta}\right) \geq 2 \quad \text { (i.e., } \operatorname{dim}|\Delta| \geq 1\right) \\ 0 & \text { otherwise, }\end{cases}
$$


where $c$ is the number of the connected components of $\left|\Delta^{\prime}\right|:=|\Delta| \backslash\{$ isolated points $\}$.

Let us prove the claim. We may assume that $\operatorname{dim}\left(R / I_{\Delta}\right)>0$. If $\operatorname{dim}\left(R / I_{\Delta}\right)=1$, then $R / I_{\Delta}$ is Cohen-Macaulay, and the assertion is clear. So we may assume that $\operatorname{dim}\left(R / I_{\Delta}\right) \geq$ 2. First, we consider the case when $I_{\Delta}$ does not have 1-dimensional associated primes, equivalently, $|\Delta|$ does not admit isolated points (i.e., $|\Delta|=\left|\Delta^{\prime}\right|$ ). Then we have

$$
\operatorname{dim}_{R}\left(\operatorname{Ext}_{R}^{n-1}\left(R / I_{\Delta}, \omega_{R}\right)\right)<1 .
$$

Since $\operatorname{Ext}_{R}^{n-1}\left(R / I_{\Delta}, \omega_{R}\right)$ is a squarefree module, we have

$$
\operatorname{Ext}_{R}^{n-1}\left(R / I_{\Delta}, \omega_{R}\right)=\left[\operatorname{Ext}_{R}^{n-1}\left(R / I_{\Delta}, \omega_{R}\right)\right]_{0} .
$$

We also have

$$
\left[\operatorname{Ext}_{R}^{n-1}\left(R / I_{\Delta}, \omega_{R}\right)\right]_{0} \cong\left[H_{\mathfrak{m}}^{1}\left(R / I_{\Delta}\right)\right]_{0} \cong \widetilde{H}^{0}(X ; \mathbb{k}) \cong \mathbb{k}^{c-1},
$$

where the second isomorphism follows from the last statement of Theorem 5.4. Hence

$$
\lambda_{0,1}\left(R / I_{\Delta}\right)=\operatorname{dim}_{\mathbb{k}}\left[\operatorname{Ext}_{R}^{n}\left(\operatorname{Ext}_{R}^{n-1}\left(R / I_{\Delta}, \omega_{R}\right), \omega_{R}\right)\right]_{0}=\operatorname{dim}_{\mathbb{k}}\left[\operatorname{Ext}_{R}^{n}\left(\mathbb{k}^{c-1}, \omega_{R}\right)\right]_{0}=c-1,
$$

and we are done.

So we now consider the case where $I_{\Delta}$ admits 1-dimensional associated primes. Set $I:=I_{\Delta^{\prime}}$. Then there is a monomial ideal $J$ of $R$ with $I_{\Delta}=I \cap J$ and $\operatorname{dim} R / J=1$. Note that $I+J=\mathfrak{m}$. The short exact sequence $0 \rightarrow R / I_{\Delta} \rightarrow R / I \oplus R / J \rightarrow R / \mathfrak{m}(\cong \mathbb{k}) \rightarrow 0$ yields the exact sequence

$$
0 \longrightarrow \operatorname{Ext}_{R}^{n-1}\left(R / I, \omega_{R}\right) \oplus \operatorname{Ext}_{R}^{n-1}\left(R / J, \omega_{R}\right) \longrightarrow \operatorname{Ext}_{R}^{n-1}\left(R / I_{\Delta}, \omega_{R}\right) \longrightarrow \mathbb{k} \longrightarrow 0 .
$$

Since Lyubeznik numbers of type $\lambda_{1,1}(-)$ are always trivial, we have

$$
\left[\operatorname{Ext}_{R}^{n-1}\left(\operatorname{Ext}_{R}^{n-1}\left(R / I_{\Delta}, \omega_{R}\right), \omega_{R}\right)\right]_{0}=\left[\operatorname{Ext}_{R}^{n-1}\left(\operatorname{Ext}_{R}^{n-1}\left(R / I, \omega_{R}\right), \omega_{R}\right)\right]_{0}=0
$$

and $\left[\operatorname{Ext}_{R}^{n-1}\left(\operatorname{Ext}_{R}^{n-1}\left(R / J, \omega_{R}\right), \omega_{R}\right)\right]_{0}=\mathbb{k}$. It is also clear that $\operatorname{Ext}_{R}^{n}\left(\operatorname{Ext}_{R}^{n-1}\left(R / J, \omega_{R}\right), \omega_{R}\right)=$ 0. Thus applying $\operatorname{Ext}_{R}\left(-, \omega_{R}\right)$ to (5.2), we obtain

$$
\begin{aligned}
0 \longrightarrow & {\left[\operatorname{Ext}_{R}^{n-1}\left(\operatorname{Ext}_{R}^{n-1}\left(R / J, \omega_{R}\right), \omega_{R}\right)\right]_{0}(\cong \mathbb{k}) \longrightarrow\left[\operatorname{Ext}_{R}^{n}\left(\mathbb{k}, \omega_{R}\right)\right]_{0}(\cong \mathbb{k}) \longrightarrow } \\
& {\left[\operatorname{Ext}_{R}^{n}\left(\operatorname{Ext}_{R}^{n-1}\left(R / I_{\Delta}, \omega_{R}\right), \omega_{R}\right)\right]_{0} \longrightarrow\left[\operatorname{Ext}_{R}^{n}\left(\operatorname{Ext}_{R}^{n-1}\left(R / I, \omega_{R}\right), \omega_{R}\right)\right]_{0} \longrightarrow 0 . }
\end{aligned}
$$

Since $\left[\operatorname{Ext}_{R}^{n}\left(\operatorname{Ext}_{R}^{n-1}\left(R / I, \omega_{R}\right), \omega_{R}\right)\right]_{0} \cong \mathbb{k}^{c-1}$ as we have shown above, it follows that

$$
\left[\operatorname{Ext}_{R}^{n}\left(\operatorname{Ext}_{R}^{n-1}\left(R / I_{\Delta}, \omega_{R}\right), \omega_{R}\right)\right]_{0} \cong \mathbb{k}^{c-1}
$$

and we are done.

Example 5.6. This example concerns the final step of the proof of Theorem 5.3. Let $R=\mathbb{k}\left[x_{1}, \ldots, x_{7}\right]$ be a polynomial ring, and consider the monomial ideal

$$
I_{\Delta}=\left(x_{2}, x_{3}, x_{4}, x_{5}, x_{6}, x_{7}\right) \cap\left(x_{1}, x_{4}, x_{5}, x_{6}, x_{7}\right) \cap\left(x_{1}, x_{2}, x_{3}, x_{6}, x_{7}\right) \cap\left(x_{1}, x_{2}, x_{3}, x_{4}, x_{5}\right) .
$$

Then $|\Delta|$ consists of 1 isolated point and 3 segments, see Fig 1 below. So $\left|\Delta^{\prime}\right|$, which is $|\Delta| \backslash\left\{v_{1}\right\}$, consists of 3 segments. We have $\lambda_{0,1}\left(R / I_{\Delta}\right)=3-1=2$. 


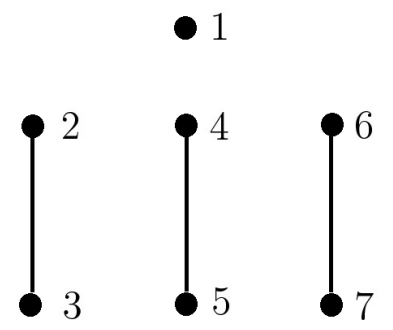

FiguRE 1.

\section{REFERENCES}

[1] J. Àlvarez Montaner and A. Vahidi, Lyubeznik numbers of monomial ideals, Trans. Amer. Math. Soc. 366 (2014), 1829-1855.

[2] J. Àlvarez Montaner, Lyubeznik table of sequentially Cohen-Macaulay rings, Comm. Algebra 43 (2015), 3695-3704.

[3] J. E. Björk, Rings of differential operators, North Holland Mathematics Library, Amsterdam, 1979.

[4] M. Blickle, Lyubeznik's numbers for cohomologically isolated singularities, J. Algebra 308 (2007), 118-123.

[5] M. Blickle, R. Bondu, Local cohomology multiplicities in terms of étale cohomology, Ann. Inst. Fourier 55 (2005), 2239-2256.

[6] R. García López and C. Sabbah, Topological computation of local cohomology multiplicities, Collect. Math. 49 (1998), 317-324.

[7] D. Helm and E. Miller, Bass numbers of semigroup-graded local cohomology, Pacific J. Math. 209 (2003), 41-66.

[8] J. Herzog and T. Hibi, Componentwise linear ideals, Nagoya Math. J. 153 (1999), 141-153.

[9] J. Herzog, Y. Takayama and N. Terai, On the radical of a monomial ideal, Arch. Math. 85 (2005), 397-408.

[10] C. Huneke and R. Y. Sharp, Bass numbers of local cohomology modules, Trans. Amer. Math. Soc. 339 (1993), 765-779.

[11] S. Jacques and M. Katzman. The Betti numbers of forests, Preprint available at arXiv:0501226.

[12] K. I. Kawasaki, On the Lyubeznik number of local cohomology modules, Bull. Nara Univ. Ed. Natur. Sci. 49 (2000), 5-7.

[13] G. Lyubeznik, Finiteness properties of local cohomology modules (an application of D-modules to commutative algebra), Invent. Math., 113 (1993) 41-55.

[14] G. Lyubeznik, F-modules: applications to local cohomology and D-modules in characteristic $p>0$, J. Reine Angew. Math. 491 (1997), 65-130.

[15] G. Lyubeznik, On some local cohomology invariants of local rings, Math. Z. 254 (2006), 627-640.

[16] W. Mahmood and P. Schenzel, On invariants and endomorphism rings of certain local cohomology modules, J. Algebra 372 (2012), 56-67.

[17] E. Miller, The Alexander duality functors and local duality with monomial support, J. Algebra 231 (2000), 180-234.

[18] M. Mustaţă, Local Cohomology at Monomial Ideals, J. Symbolic Comput. 29 (2000), 709-720.

[19] R. Okazaki and K. Yanagawa, Linearity defects of face rings, J. Algebra 314 (2007), 362-382.

[20] T. Römer, On minimal graded free resolutions, PhD. Thesis, Essen (2001).

[21] P. Schenzel, On the structure of the endomorphism ring of a certain local cohomology module, J. Algebra 344 (2011), 229-245. 
[22] R. P. Stanley, Combinatorics and Commutative Algebra, Second Edition, Progress in Mathematics 41, Birkhäuser, 1996.

[23] N. Terai, Local cohomology modules with respect to monomial ideals, Preprint 1999

[24] U. Walther, On the Lyubeznik numbers of a local ring, Proc. Amer. Math. Soc., 129 (2001), 16311634.

[25] K. Yanagawa, Alexander duality for Stanley-Reisner rings and squarefree $\mathbb{N}^{n}$-graded modules, J. Algebra 225 (2000), 630-645.

[26] K. Yanagawa, Bass numbers of local cohomology modules with supports in monomial ideals, Math. Proc. Cambridge Philos. Soc. 131 (2001), 45-60.

[27] K. Yanagawa, Sheaves on finite posets and modules over normal semigroup rings, J. Pure Appl. Algebra 161 (2001), 341-366.

[28] K. Yanagawa, Stanley-Reisner rings, sheaves, and Poincaré-Verdier duality, Math. Res. Lett. 10 (2003) 635-650.

[29] K. Yanagawa, Derived category of squarefree modules and local cohomology with monomial ideal support, J. Math. Soc. Japan 56 (2004), 289-308.

[30] K. Yanagawa, Notes on $C$-graded modules over an affine semigroup ring $K[C]$, Comm. Algebra, 36 (2008), 3122-3146.

[31] W. Zhang, On the highest Lyubeznik number of a local ring, Compos. Math. 143 (2007), 82-88.

Departament de Matemàtiques, Universitat Politècnica de Catalunya, Av. Diagonal 647, Barcelona 08028, SPAIN

E-mail address: Josep.Alvarez@upc.edu

Department of Mathematics, Kansai University, Suita 564-8680, Japan

E-mail address: yanagawa@ipcku.kansai-u.ac.jp 